\title{
Ring Galaxies in the EAGLE Hydrodynamical Simulations
}

\author{
Ahmed Elagali ${ }^{1,2 \star}$, Claudia D. P. Lagos ${ }^{1,2}$, O. Ivy Wong ${ }^{1,2}$, Lister Staveley-Smith ${ }^{1,2}$, \\ James W. Trayford ${ }^{3,4}$, Matthieu Schaller ${ }^{3,4}$, Tiantian Yuan ${ }^{2,5}$, Mario G. Abadi ${ }^{6,7}$ \\ ${ }^{1}$ International Centre for Radio Astronomy Research (ICRAR), M468, The University of Western Australia, 35 Stirling Highway, Crawley, WA 6009, Australia \\ ${ }^{2}$ ARC Centre of Excellence for All Sky Astrophysics in 3 Dimensions (ASTRO 3D) \\ ${ }^{3}$ Leiden Observatory, Leiden University, PO Box 9513, NL-2300 RA Leiden, the Netherlands \\ ${ }^{4}$ Department of Physics, Institute for Computational Cosmology, University of Durham, South Road, Durham DH1 3LE, UK \\ ${ }^{5}$ Centre for Astrophysics and Supercomputing, Swinburne University of Technology, Hawthorn, Victoria 3122, Australia \\ ${ }^{6}$ Instituto de Astronomía Teórica y Experimental, CONICET-UNC, Laprida 854, X5000BGR, Córdoba, Argentina \\ ${ }^{7}$ Observatorio Astronómico de Córdoba, Universidad Nacional de Córdoba, Laprida 854, X5000BGR, Córdoba, Argentina
}

Accepted 00. Received 00; in original form 00

\begin{abstract}
We study the formation and evolution of ring galaxies in the Evolution and Assembly of GaLaxies and their Environments (EAGLE) simulations. We use the largest reference model Ref-L100N1504, a cubic cosmological volume of 100 comoving megaparsecs on a side, to identify and characterise these systems through cosmic time. The number density of ring galaxies in EAGLE is in broad agreement with the observations. The vast majority of ring galaxies identified in EAGLE (83 per cent) have an interaction origin, i.e., form when one or more companion galaxies drop-through a disk galaxy. The remainder ( 17 per cent) have very long-lived ring morphologies ( $>2 \mathrm{Gyr}$ ) and host strong bars. Ring galaxies are HI rich galaxies, yet display inefficient star formation activity and tend to reside in the green valley particularly at $z \gtrsim 0.5$. This inefficiency is mainly due to the low pressure and metallicity of their interstellar medium (ISM) compared with the ISM of similar star-forming galaxies. We find that the interaction(s) is responsible for decreasing the ISM pressure by causing the ISM gas to flow from the inner regions to the outer disk, where the ring feature forms. At a fixed radius, the star formation efficiency of ring galaxies is indistinguishable from their star-forming counterparts, and thus the main reason for their integrated lower efficiency is the different gas surface density profiles. Since galaxy morphologies are not used to tune the parameters in hydrodynamical simulations, the experiment performed here demonstrates the success of the current numerical models in EAGLE.
\end{abstract}

\section{Key words:}

cosmology: theory - galaxies: structure - galaxies: statistics - galaxies: formation - galaxies: haloes - methods: numerical - galaxies: starburst.

\section{INTRODUCTION}

Galaxy morphology is strongly correlated with the physical properties of galaxies such as their star formation histories and dynamical structures, and hence provides crucial insights into galaxy formation and is a key diagnostic of their evolution. In the local Universe, galaxies are categorised into three main morphological types, i.e., spirals, spheroids, and irregular dwarf systems (Hubble 1926, the Hubble sequence). Observations of galaxies reveal that a considerable fraction $(\sim 10$ per cent $)$ of galaxies in the local Universe have irregular morphological structures and do not fit within the conventional Hubble classification (Nair \&

^ E-mail ahmed.elagali@icrar.org
Abraham 2010; Baillard et al. 2011; Willett et al. 2013). These morphologically disturbed systems are usually undergoing interactions and/or mergers with neighbouring galaxies (Toomre 1977; White 1978; Barnes \& Hernquist 1996; Naab et al. 2006, 2014; Rodriguez-Gomez et al. 2017). One of the most peculiar systems in our local Universe are the so-called ring galaxies. Ring galaxies are generally divided into two sub-classes, the $\mathrm{P}$ and O-types (Few \& Madore 1986). P-type rings result from an off-centre passage of a compact companion galaxy, the intruder, through the disk of much more massive spiral galaxy, the target, see for example Lynds \& Toomre (1976); Theys \& Spiegel (1977); Struck-Marcell \& Lotan (1990). This type of collision produces a density wave that radially transports the gas and stars into a ring morphology throughout the disk of the target galaxy (Appleton \& Struck-Marcell 1996; 
Mayya et al. 2005; Wong et al. 2006; Romano et al. 2008; Fogarty et al. 2011; Parker et al. 2015; Conn et al. 2016; Elagali et al. 2018). Depending on the collision's geometry, the target's nucleus can be displaced off its dynamical centre, the whole system (ring and nucleus) can be slightly dislocated from the previous plane of the disk (Mapelli \& Mayer 2012) and in some extreme cases the nucleus can completely be disrupted by the collision, producing a centrally smoothed ring with no apparent nucleus (Madore et al. 2009). The O-type are the resonance ring galaxies (de Vaucouleurs 1959; Herrera-Endoqui et al. 2015; Buta 2017). These rings are preferentially found in barred galaxies, with the ring encircling the bar and forming the familiar $\theta$-shape. The O-type rings are not the products of violent galaxy collisions, but rather are formed by gas accumulation at Lindblad resonances under the continuous influence of gravity torques from the bars.

Collisional ring galaxies (P-type) have been widely studied in idealised (isolated) interaction simulations, in which a companion galaxy collides with a disk galaxy using certain interaction parameters to produce the ring morphology (Lynds \& Toomre 1976; Theys \& Spiegel 1977; Struck-Marcell \& Lotan 1990; Hernquist \& Weil 1993; Gerber et al. 1996; Athanassoula et al. 1997; Horellou \& Combes 2001; Mapelli et al. 2008b; Smith et al. 2012; Mapelli \& Mayer 2012; Renaud et al. 2018). These simulations show that certain impact parameters and collision angles can induce warps in the disk, affect the morphology and the star formation history of the target disk galaxy (Fiacconi et al. 2012). Mihos \& Hernquist (1994) and Mapelli et al. (2008a) showed in their simulations that it takes around $100 \mathrm{Myr}$ after the collision to develop the ring in the disk of these galaxies and that the ring morphology remains visible for $\sim 0.5 \mathrm{Gyr}$, which is very short compared to the Hubble timescale. Since the formation mechanisms of collisional ring galaxies are very well constrained via simulations, they can be considered as galaxy-scale perturbation experiments that facilitate the study of extreme modes of interaction-triggered star formation and feedback processes (Higdon et al. 2012, 2015; Wong et al. 2017; Renaud et al. 2018).

Even though non-cosmological isolated interaction simulations are cornerstones in understanding the formation mechanisms of collisional ring galaxies, they lack the statistical basis that would allow for a systematic study of these galaxies in the local Universe. This is because these simulations consist of only the target and the intruder companion, using arbitrary initial conditions and collision parameters to induce the ring morphology in the target galaxy. Hence, it is important to expand the sample of simulated collisional ring galaxies and study larger volumes that cosmologically represent the local Universe. This will advance our understanding of the collisional ring galaxies' number density, their evolution and whether the drop-through interaction proposed in non-cosmological isolated interaction simulations is frequent enough to explain the observed number density of ring galaxies (Lavery et al. 2004; Elmegreen \& Elmegreen 2006). Further, observations of collisional ring galaxies suggests that these systems contain on average high amounts of HI gas in comparison with galaxies that have the same stellar mass (Elagali et al. 2018), yet are $\mathrm{H}_{2}$ deficient (Higdon et al. 2015; Wong et al. 2017) especially at the outer rings where the atomic hydrogen surface density is the highest (e.g., in the Cartwheel galaxy $\Sigma_{H I}=19-65 \mathrm{M}_{\odot} \mathrm{pc}^{-2}$; Higdon et al. 2015). The reason behind this deficiency is not yet well understood, however Wong et al. (2017) and Higdon et al. (2015) hypothesise that the ISM in ring galaxies behaves differently as a result of the extreme conditions in, e.g., pressure, temperature and metallicity, induced by the drop-through collision.

In their N-body cosmological simulation, D'Onghia et al. (2008) adopted a method to identify progenitor merging haloes that host collisional ring galaxies based on their halo masses, the mass ratio between the two merging haloes, and the the impact parameter of the collision (the initial distance vector between the centre of mass of the two merging haloes). They used the resultant number density of collisional ring galaxies throughout cosmic time to place constraints on the merger rate. But it is unclear how biased rings are as tracers of interactions and whether that bias is tim1990Strucke invariant or not. A more recent study of ring galaxies in a cosmological hydrodynamical dynamical simulation was conducted by the Illustris team (Snyder et al. 2015). They explored the morphology of galaxies in these simulations and found that at $z=0$ a considerable fraction of galaxies within the mass range $10^{10.5}-10^{11} \mathrm{M}_{\odot}$ have a distinct ring/C-shape morphology. However, the abundance of these systems in their simulations is much higher than observational studies suggest. One possible reason for this over population of ring galaxies in Illustris is the choice of interstellar medium (ISM) and feedback models. While this is still inconclusive, in the new Illustris project (Illustris-TNG; Pillepich et al. 2017; Springel et al. 2017; Naiman et al. 2017; Marinacci et al. 2017; Nelson et al. 2017) ring-like structures are not as abundant, which suggests that the problem with Illustris is likely a combination of effects from different subgrid physics modules. This is because in Illustris-TNG the stellar and AGN feedback models are implemented differently in comparison to Illustris, which possibly affects the way gas piles up in galaxies and consequently their morphology (Weinberger et al. 2017; Pillepich et al. 2017).

Here, we intend to study collisional ring galaxies in the EAGLE simulations, trace the abundance of these systems with redshift, compare it with observations, and study their formation mechanisms. The EAGLE simulations, which stands for Evolution and Assembly of GaLaxies and their Environments (Schaye et al. 2015; Crain et al. 2015), reproduces many key observational results such as the specific star formation rates (Furlong et al. 2015), the passive galaxy fraction (Trayford et al. 2015), the Tully-Fisher relation (Schaye et al. 2015), the $\mathrm{H}_{2}$ mass functions at $z=0$ and several atomic and molecular gas scaling relations (Lagos et al. 2015; Bahé et al. 2016; Crain et al. 2017). The fact that we can detect ring galaxies in a statistical sample of simulated galaxies implies that we can use these systems as a higher-order constraint on the models because morphology was not used to tune the parameters in EAGLE (see Crain et al. (2015) for more details). Thus, our study represents a true prediction of the simulation and by comparing these results with the observations we hope to learn whether the numerical treatments of the ISM, star formation, and feedback are adequate enough to reproduce a realistic ringmorphology population. We also study the general characteristics of collisional ring galaxies in the EAGLE simulations including their star formation rates (SFRs), colours, metallicities, atomic $(\mathrm{HI})$ and molecular hydrogen $\left(\mathrm{H}_{2}\right)$ gas scaling relations. We aim to provide a thorough description of the ISM properties of collisional ring galaxies in the EAGLE simulations and the possible reasons leading to their high $\mathrm{HI}$ masses or molecular gas deficiencies.

This paper is organised as follows: In Section 2, we briefly describe the key characteristics of the simulation, the subgrid physics 
included in EAGLE and the mock optical images generated by the SKIRT code (Trayford et al. 2017, hereafter T17). Section 3 describes our selection criteria applied to select ring galaxies in the simulation. In section 4 , we present the characteristics of ring galaxies in EAGLE, including their number density, halo masses and the concentrations of the haloes containing rings, colour-magnitude diagram, HI-stellar mass relation as well as the SFR-stellar mass of ring galaxies, at different redshifts. We also present a more detailed study of the ISM properties in ring galaxies. In Section 5 we present the origin and the formation history of rings in the EAGLE simulation. We present our discussion and conclusion in Section 6. For all the calculations, we adopt the cosmology used for the EAGLE simulation, a $\Lambda$ CDM cosmology with $\Omega_{m}=0.307, \Omega_{\Lambda}=0.693, \Omega_{b}=0.048, \sigma_{8}=0.8288$, and $H_{0}=67.77 \mathrm{~km} \mathrm{~s}^{-1} \mathrm{Mpc}^{-1}$, consistent with Planck measurements (Planck Collaboration et al. 2014).

\section{SIMULATED GALAXY SAMPLES}

\subsection{Overview of the EAGLE Simulation}

The EAGLE project is a suite of hydrodynamical simulations designed to explore the evolution of the Universe's baryonic matter (gas, stars and massive black holes) and dark matter from a redshift of $z=127$ until $z=0$ (Schaye et al. 2015; Crain et al. 2015). The simulations were constructed assuming the standard cosmological paradigm (the so called $\Lambda$ CDM cosmological model) and run with an extensively modified version of the N-body TreePM smoothed particle hydrodynamics (SPH) code GADGET 3 (Springel 2005; Springel et al. 2008). The main updates to the standard GADGET 3 code feature modifications to the hydrodynamics algorithm, and the incorporation of subgrid modules that capture the unresolved physics acting on scales below the resolution limit of the simulations. The modified SPH algorithm, referred to as 'Anarchy', includes the implementation of the pressure-entropy formulation of SPH (Hopkins 2013), the artificial viscosity switch proposed by Cullen \& Dehnen (2010), the artificial conduction switch similar to that proposed by Price (2008), the $\mathrm{C}_{2}$ smoothing kernel of Wendland (1995), and the timestep limiter from Durier \& Dalla Vecchia (2012). The impact of the above modifications to the standard GADGET 3 code and its effect on the simulated galaxies is described in Schaller et al. (2015).

The EAGLE simulations incorporate state-of-the-art subgrid physics based on those used for the OWLS (Schaye et al. 2010) and the GIMIC (Crain et al. 2009) projects. These subgrid physics models include element-by-element radiative cooling and photoheating rates (Wiersma et al. 2009a), star formation as a pressure law (Schaye \& Dalla Vecchia 2008) and a metallicity-dependent density threshold (Schaye 2004), stellar evolution and elementby-element chemical enrichment (Wiersma et al. 2009b), stellar feedback as energy injected from core-collapse supernovae (Dalla Vecchia \& Schaye 2012), as well as accreting black holes (BH) and AGN feedback (Rosas-Guevara et al. 2015). The efficiency of the stellar feedback and the $\mathrm{BH}$ accretion were calibrated to match the observations of the galaxy stellar mass function (GSMF) at $z=0.1$, while the AGN feedback was calibrated to match the observed relation between stellar mass and BH mass. The EAGLE simulation has had unprecedented successes as it reproduces many key observational datasets (that were not considered during
Table 1. Key parameters of the EAGLE Ref-L100N1504 simulation used in this paper. The EAGLE simulation adopts a softening length of $2.66 \mathrm{ckpc}$ at $z \geq 2.8$, and $0.7 \mathrm{pkpc}$ at $z<2.8$.

\begin{tabular}{ll}
\hline \hline Comoving box size & $100 \mathrm{cMpc}$ \\
Number of particles & $2 \times 1504^{3}$ \\
Gas particle mass & $1.81 \times 10^{6} \mathrm{M}_{\odot}$ \\
Dark matter particle mass & $9.7 \times 10^{6} \mathrm{M}_{\odot}$ \\
Softening length & $2.66 \mathrm{ckpc}$ \\
Max. gravitational softening length & $0.7 \mathrm{pkpc}$ \\
\hline
\end{tabular}

the calibration) including the stellar mass function of galaxies (Furlong et al. 2015), the stellar mass-size relation (Furlong et al. 2017), the colour distribution of galaxies (Trayford et al. 2015), the cold gas contents of galaxies throughout cosmic time (Lagos et al. 2015, 2016; Bahé et al. 2016; Crain et al. 2017), and the evolution of the star formation rate with redshift (Katsianis et al. 2017), among others.

For this work, we use the largest reference model RefL100N1504 (hereafter Ref-100), which is a cubic cosmological volume of 100 comoving megaparsecs (cMpc) on a side, with dark matter particle mass of $9.7 \times 10^{6} \mathrm{M}_{\odot}$, initial gas particle mass of $1.81 \times 10^{6} \mathrm{M}_{\odot}$, and equal numbers of baryonic and dark matter particles $\left(1504^{3}\right)$. The Plummer equivalent gravitational softening lengths are set to $1 / 25$ of the initial mean interparticle spacing and are 0.7 proper kiloparsecs (pkpc) at redshifts $z<2.8$, and 2.66 comoving kiloparsecs (ckpc) at earlier times, which means that the Jeans scales in the warm ISM are marginally resolved. The volume of Ref-L100N1504 provides a wide range of galaxy morphologies in a sample of $\sim 30,000$ galaxies resolved by $>1,000$ star particles and $\sim 3,000$ galaxies resolved by $>10,000$ star particles at redshift $z=0.1$. Table 1 presents a summary of the key features of the reference model Ref-L100N1504. The properties of the particles in the simulation were recorded for 29 snapshots between redshifts 20 and 0 , which translates to time span range between snapshots of $0.3-1 \mathrm{Gyr}$. However, finer time resolution are also available, referred to as 'snipshots' in Crain et al. (2017), in which a smaller set of particle properties are saved at 400 redshifts between $20 \leq z \leq 0$. It is important to note that only 200 of the 400 snipshots were used to construct the merger trees; the merger trees are computationally expensive to construct. Hence, the time resolution of the snipshots span between $0.05-0.3 \mathrm{Gyr}$. We use the merger trees available in the EAGLE database (McAlpine et al. 2016) to trace the evolution of ring galaxies in the snapshots of the simulation, while for snipshots we use the private merger trees catalogue. All these merger trees were constructed as in Qu et al. (2017).

\subsection{Mock observations \& Images}

Camps et al. (2016); Trayford et al. (2017, hereafter T17) present a novel method to generate mock synthetic optical images of galaxies in the EAGLE simulations that includes the effects of dust using the SKIRT Monte Carlo radiative transfer code (Baes et al. 2003, 2011; Camps \& Baes 2015). T17 compute a full spectral energy distribution (SED) for each star particle using the the GALAXEV population synthesis models of Bruzual \& Charlot (2003), taking into account the stellar ages, smoothed metallicities and initial masses of the star particles. They also use the MAPPINGS photoionisation code (Groves et al. 2008) to 

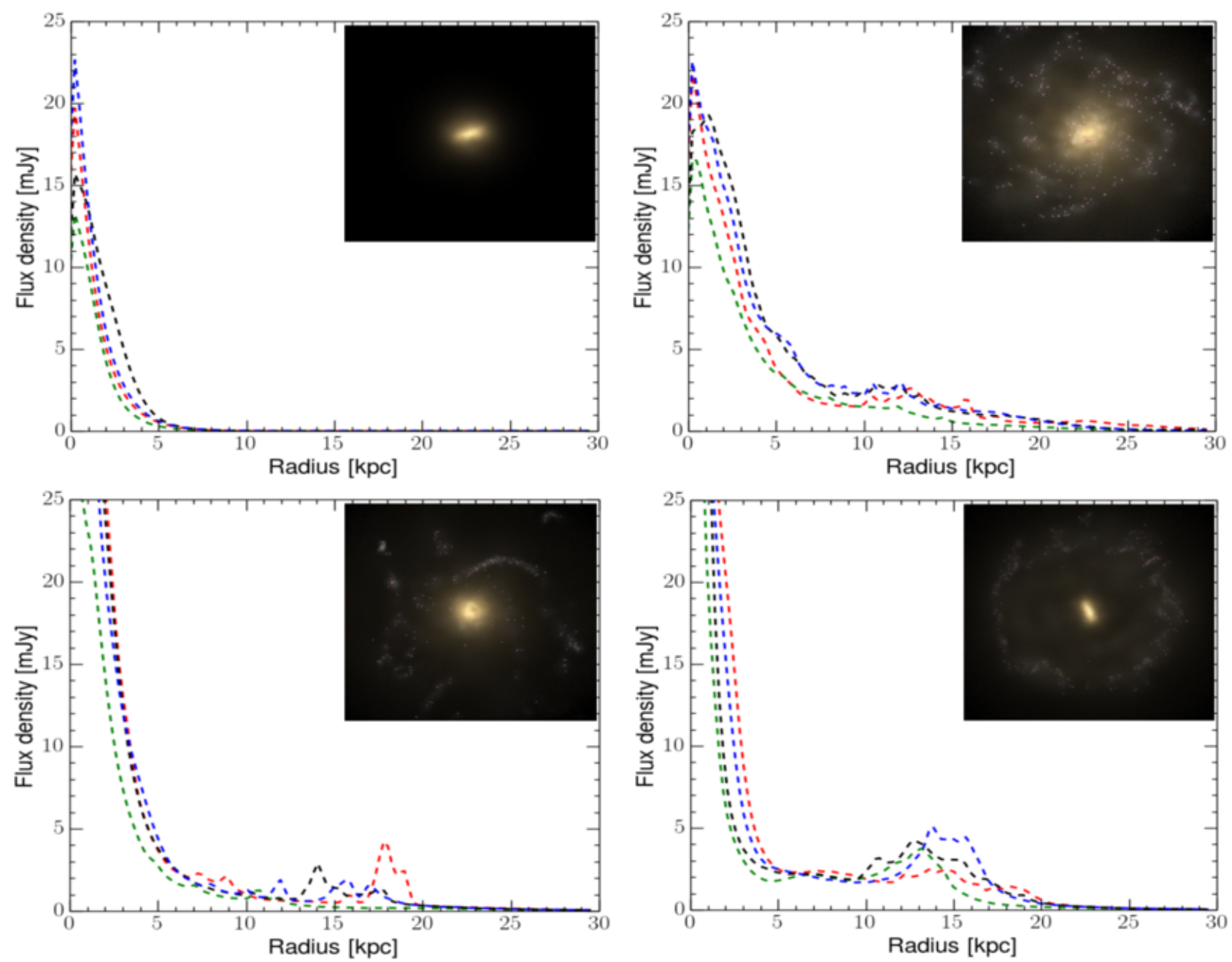

Figure 1. The kernel smoothed radial flux profile in four different strips (shown by the coloured dashed-lines) of the face-on $u$-band image of an elliptical (top left), spiral (top right), and two morphologically disturbed (interacting; bottom panels) galaxies at $z=0$. The radial profiles describe the flux distribution of that respected galaxy from its centre to its outer edge along its major and minor axes. The inset shows the synthetic gri composite image of these galaxies. Only one of the two interacting galaxies is classified as a ring galaxy (bottom right).

describe the effects of dust associated with star-forming regions, nebular line and continuum spectra throughout the HII regions. The SKIRT Monte Carlo code then uses these sets of sources and dust distributions to determine the path of the monochromatic photons (absorption \& scattering) until they hit the user specified detector.

T17 construct images in ugriz SDSS bands (Doi et al. 2010) of 3,624 galaxies with $\mathrm{M}_{*}>10^{10} \mathrm{M}_{\odot}$ in the EAGLE hydrodynamical simulation at redshift $z=0.1$. These observations are available in three orientations: edge on, face on and randomly orientated with respect to the galactic plane. Each image is $256 \times 256$ pixels in size, with field of view (FOV) of $60 \mathrm{kpc}$, and with the detector camera placed $50 \mathrm{Mpc}$ away from the galaxy centre. The mock observations are performed as if each galaxy is in isolation, hence disregard all the contribution from other sources and structures along the line of sight or closer than $60 \mathrm{kpc}$ in projection.

\section{SELECTION OF RING GALAXIES IN EAGLE}

We develop a Python algorithm specifically designed to identify ring galaxies in the Ref-100 simulation box. The routine makes use of the face-on orientation ugriz-images created by T17 and broadly quantifies galaxy morphologies into ellipticals and lenticulars (early-type), spirals (late-type), and morphologically disturbed (interacting) systems. This algorithm relies on the radial flux profiles of these galaxies and works as following:

- Each galaxy is divided into four strips, each $4 \mathrm{kpc}$ in width, along the major and the minor axis of the galaxy. For each strip, we construct a radial flux profile using the rest-frame face-on $u$-band images, and smooth this profile using a kernel function with a Gaussian scale. By definition each galaxy is placed at the centre of image, hence the radial profile describes the flux distribution of each respective galaxy from its centre to its outer edge. For early-type (elliptical and lenticular) galaxies, the flux distribution varies rapidly with radius with a high peak at the centre of the galaxy that decreases abruptly with radius. The case is slightly different for late-type (spiral) galaxies, where the flux decreases gradually with radius and the spiral arms produce flux increments 


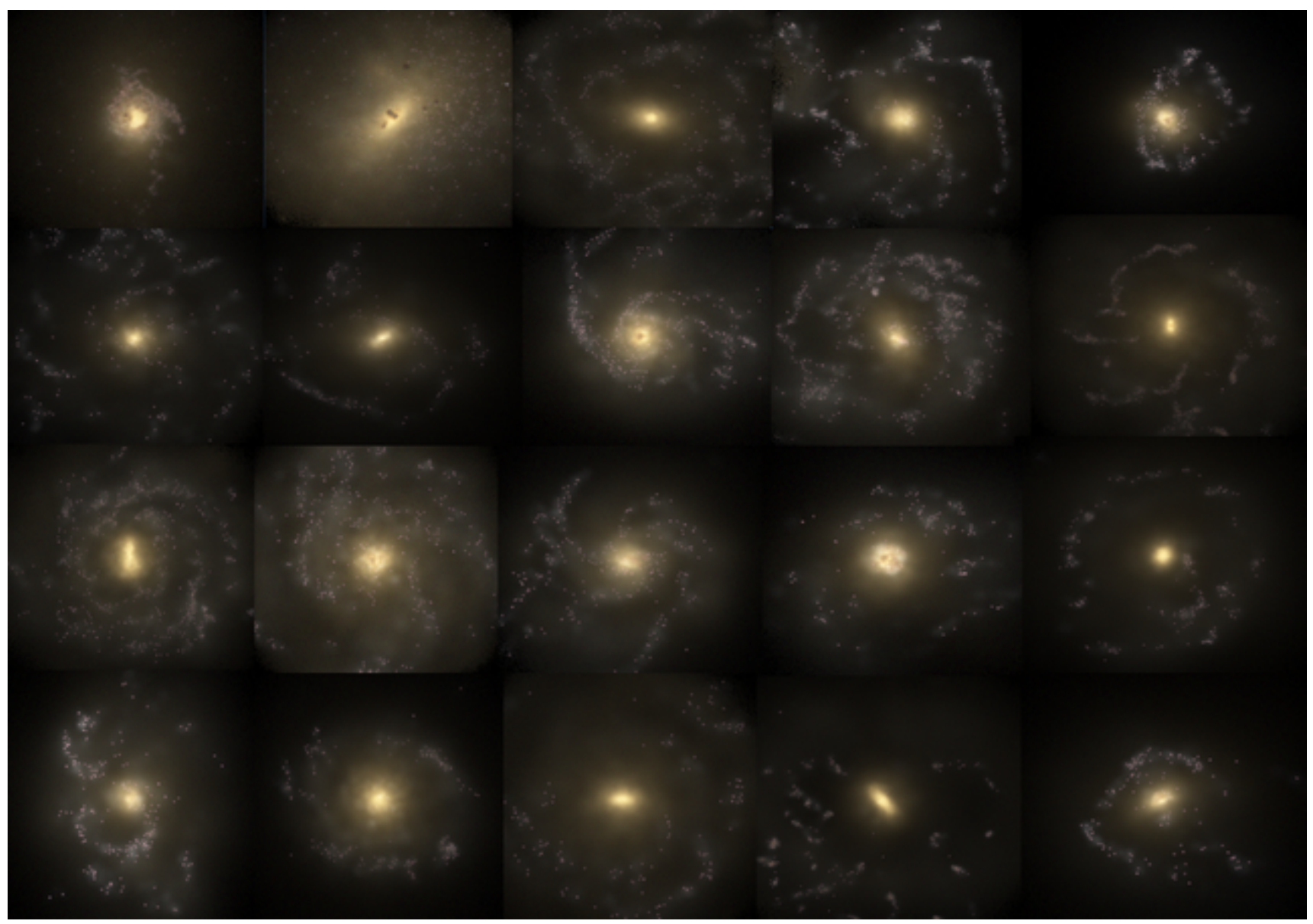

Figure 2. Three-colour gri mock images of a subsample of the interacting galaxy candidates selected by the algorithm at $z=0$. The majority of these galaxies are morphological disturbed or interacting systems and a small fraction of these systems are real ring galaxies (far right column). These images are $60 \mathrm{kpc}$ on a side and are publicly available from the EAGLE database (McAlpine et al. 2016).

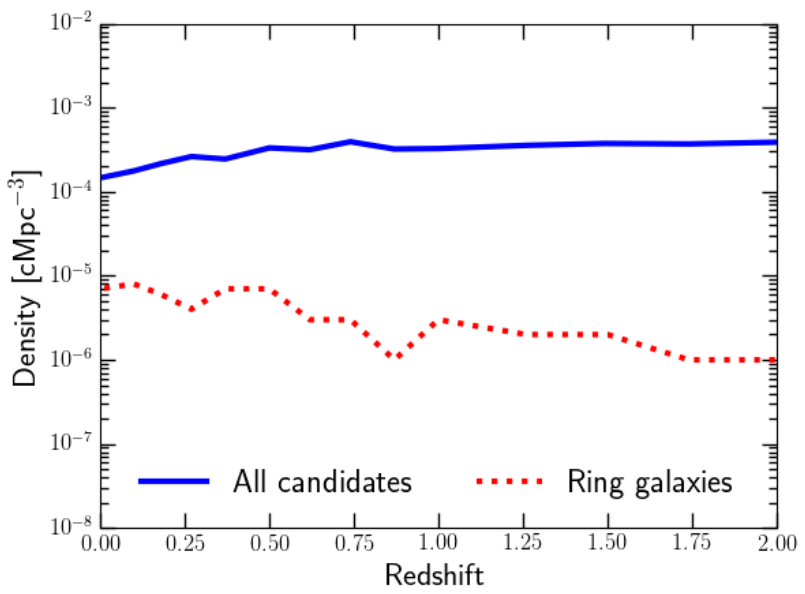

Figure 3. The number density of the interacting or disturbed candidates against redshift (blue line) and the visually selected subsample of ring or C-shape morphology candidates (red dotted line). The number of interacting galaxy candidates increases with redshift in agreement with the interactions/mergers rates (refer to Figure 4). (bumps) throughout the radial flux profile. The radial profile of interacting (morphologically disturbed) galaxies is similar to that of the late-type galaxies, albeit the bumps are more extended and the flux increments are higher. It is important to note that no changes to the final results will occur if the width of the strip is slightly larger than $4 \mathrm{kpc}$.

- Next the algorithm searches and quantifies the flux increments (bumps) in the radial profile of each galaxy and uses both the extent (width) and the amount of flux boost in each bump as a broad indicator to quantify the galaxy morphology. Early-type galaxies are the simplest to segregate, in which there are no flux increments with radius in the profiles and the flux decreases smoothly with increasing radius. To distinguish between late-type and morphologically disturbed systems, we use the width of the bump and the flux increment present in the galaxy radial profile. Galaxies with continuous bump(s) that extends for $\gtrsim 2 \mathrm{kpc}$ in at least two strips and the flux boost in each bump is $\geq 10 \%$ are considered as interacting candidates. Figure 1 presents an example of the kernel smoothed radial flux distribution in four strips of the face-on image of an elliptical, spiral, and two morphologically disturbed (interacting) galaxies at redshift $z=0$, respectively. The inset shows the $u$-band image of the same galaxies. The flux distribution in the elliptical galaxy has no bumps and decreases continuously with increasing radius. On the other hand, the radial 

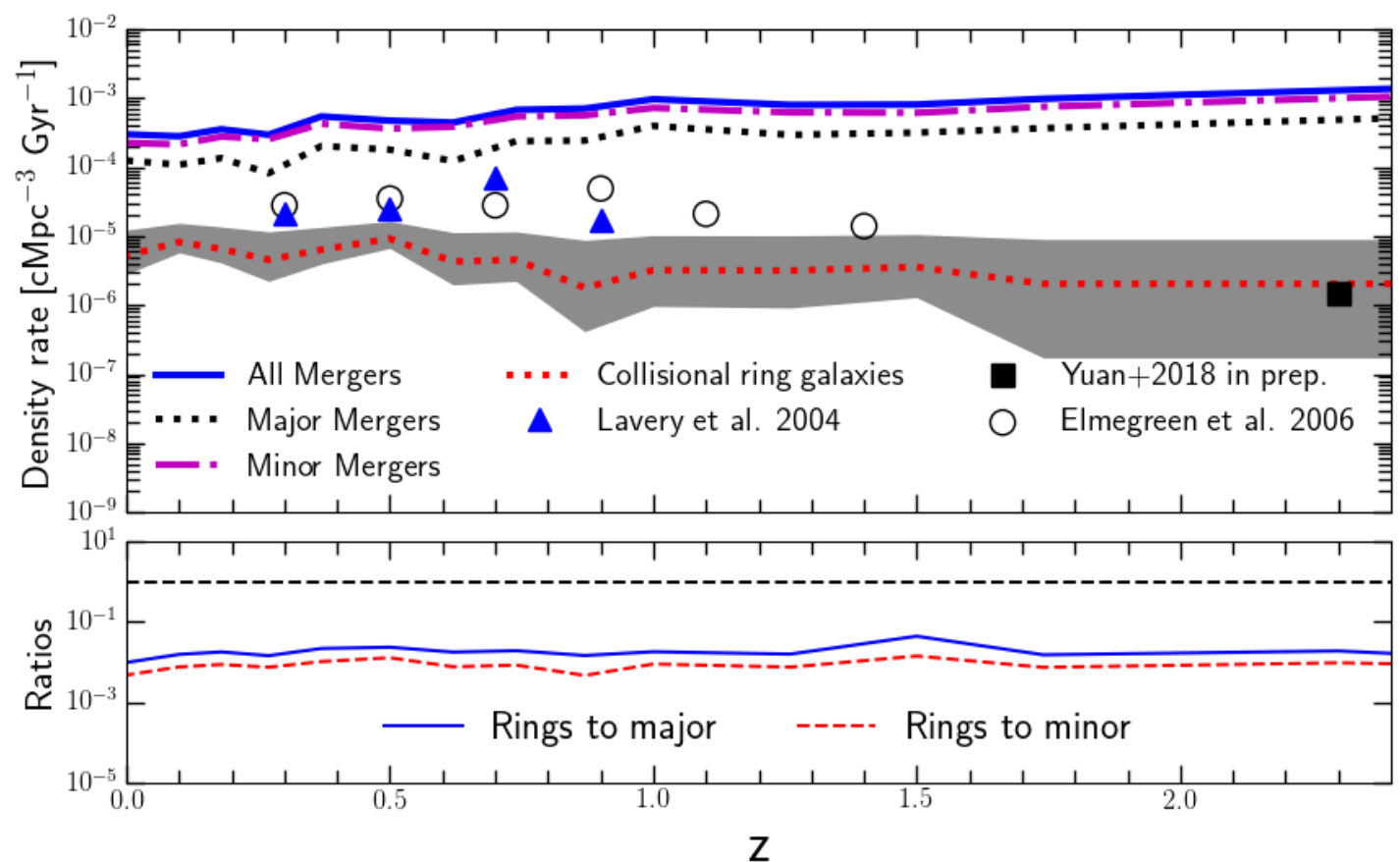

Figure 4. Upper Panel: The formation density rate of ring galaxies in the EAGLE simulations (red dotted line) compared to the number density from observations (black unfilled circles from Elmegreen \& Elmegreen 2006, blue triangles from Lavery et al. 2004, and filled square from Yuan et al. 2018 in prep.). The grey shaded region marks the Poisson errors in the EAGLE number density. The blue line shows the number density of all mergers in the simulation with a stellar mass ratio $\gtrsim 0.1$, while the black dotted and magenta dash-dotted lines show the contribution from major (mass ratios $\gtrsim 0.3$ ) and minor mergers (mass ratios between $0.1-0.3$ ), respectively. EAGLE agrees very well with the ring galaxy observations. Lower Panel: The ratio between the collisional ring galaxies number density to the major (blue line) and minor (red dashed line) merger rates. The black dashed line represents equality.

flux profile in the spiral and the interacting candidates shows many bumps with increasing radius. However, the difference between the two is that the spiral galaxy shows less boost in the flux at each bump $(<10 \%)$ compared with the two interacting systems in the bottom panel.

- Lastly, the algorithm makes a list of all the interacting candidates and creates plots similar to Figure 1 for those galaxies. The final step is to visually inspect the interacting galaxies subsample and only select galaxies that have a ring or C-shape morphology. Visual inspection is necessary in order to distinguish the P-type (collisional) and resonant O-type ring galaxies and to determine the presence of any weak bar-like or spiral structure. Figure 2 shows a subsample of the gri mock images of the interacting galaxy candidates selected by the algorithm at $z=0$, the majority of these galaxies are morphologically disturbed or interacting systems and a smaller fraction are the real ring galaxies. In this figure the true ring galaxies are located at the far right column. We visually inspect all the interacting galaxy candidates generated by the algorithm between redshift $z=0$ to $z=2$ and select only the candidates with a ring or $\mathrm{C}$-shape morphology. Figure 3 shows the number density of the interacting or disturbed candidates as a function of redshift (blue line) along with the visually selected subsample of ring or C-shape morphology candidates (red dotted line). The number of disturbed or potentially interacting galaxies increases with redshift, which is consistent with other studies (e.g., Abraham et al. 1996; Fakhouri et al. 2010; Bluck et al.
2012; Man et al. 2016). The total number of interacting candidates varies between 150 at redshift $z=0$ up to 350 candidates at $z=2$.

To investigate the success rate of our algorithm, we search for ring galaxies visually at redshifts $z=0,0.5$ and 1.0 using the gri composite images available in the database (McAlpine et al. 2016). We inspect all the galaxies in these three snapshots that have a stellar mass of $\mathrm{M}_{*} \geq 10^{10} \mathrm{M}_{\odot}$ (corresponding to the typical masses of observed ring galaxies) and record all of the ring galaxies in these snapshots. Then, we run this semi-automated algorithm and compare the visually inspected ring galaxies with the the list of interacting candidates that results at redshifts $z=0,0.5$ and 1.0. We find that the list of interacting candidates generated by the semi-automated algorithm recovers all the ring galaxies that were visually found. Further, to test the reliability of the algorithm, we set the extent of the bump to $3 \mathrm{kpc}$ and $4 \mathrm{kpc}$ instead of $2 \mathrm{kpc}$. The number of candidates in the former two cases are similar to the number of candidates that results from setting the required extent to $2 \mathrm{kpc}$. However, if the extent of the bump decreases to $1 \mathrm{kpc}$ or less, the number of candidates increases by up to $20 \%$ more at a given redshift. The majority of the new candidates are spiral galaxies, with no real ring galaxies. Hence, setting the width of the bump to $2 \mathrm{kpc}$ is optimal and reduces the number of false candidates appearing as a result of the spiral arms in late type galaxies. Further, we apply our algorithm to the EAGLE higher resolution smaller box Ref-L025N0752 (Schaye et al. 2015) and search for ring galaxies between redshifts $z=0$ to $z=2$, 

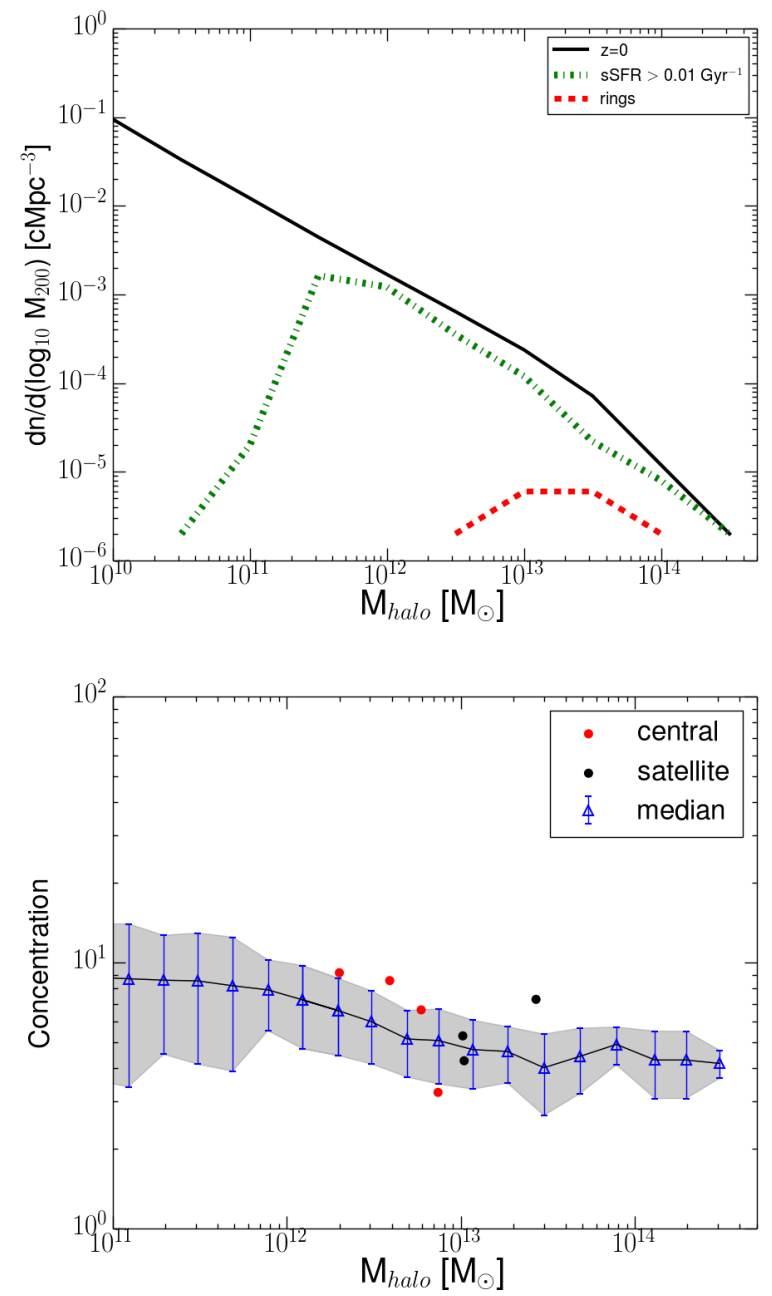

Figure 5. Upper Panel: The halo mass function for all the haloes at $z=0$ (black line), and for the subsample of haloes hosting star-forming central galaxies with sSFR $>0.01 \mathrm{Gyr}^{-1}$ (green dash-dotted line) and ring galaxies (red dashed line). Lower Panel: The concentration of the haloes as a function of their mass at $z=0$. The grey shaded region shows the $68^{\text {th }}$ percentile while the median is shown as blue triangles. The red and black symbols are the concentrations of haloes hosting central and non-central ring galaxies, respectively.

with the aim of uncovering any obvious resolution effects on the formation process of ring galaxies. We find no ring galaxies in this simulation box, which agrees with the expected number density of ring galaxies from the observations. In Ref-L025N0752 box $\left(25^{3}\right.$ $\mathrm{cMpc}^{3}$ ), the expected number of ring galaxies in each snapshot is $<0.06$, using the number density of $4.3 \times 10^{-6} \mathrm{Mpc}^{-3}$ from Lavery et al. (2004). This result provides no evidence to suggest that the ring galaxies detected in Ref-100 are due to resolution effects.

To distinguish between the $\mathrm{P}$ and O-type ring galaxies, we crossmatch our sample with the sample of barred galaxies in EAGLE reported in Algorry et al. (2017, hereafter A17). A17 analyse the central galaxies in EAGLE that lie within mass range of $10.6<\log _{10}\left(\mathrm{M}_{*} / \mathrm{M}_{\odot}\right)<11$ at redshift $z=0$ and classify galaxies via the amplitude of the normalised $m=2$ Fourier mode of the azimuthal distribution of their disk particles. Galaxies with a normalised amplitude $\left(\mathrm{A}_{2}^{\max }\right)<0.2$ are unbarred systems, those with an amplitude in the range of $0.2<\mathrm{A}_{2}^{\max }<0.4$ have a weak bar, while strongly barred galaxies have an amplitude $\mathrm{A}_{2}^{\max }>0.4$. At redshift $z=0$, we identify seven systems as ring galaxies, one of which has a strong bar $\left(\mathrm{A}_{2}^{\max }>0.4\right)$ and the remainder are unbarred galaxies. At higher redshift, we explore the formation history of our ring galaxy sample to distinguish between barred and collisional ring galaxies. This is discussed in Section 5.

\section{CHARACTERISATION OF THE RING GALAXY POPULATION IN EAGLE}

In this section, we study the number density, environment and properties of ring galaxies. We pay special attention to the relation between the gas abundance and star formation in the ring galaxies to understand the origin of their star formation deficiency.

\subsection{Rings as Tracers of Galaxy Mergers}

The upper panel of Figure 4 shows the formation density rate of collisional ring galaxies identified in the EAGLE simulations (red dash-line) and in observations as a function of redshifts. The grey shaded region marks the one-sigma scatter in the EAGLE number density rate ${ }^{1}$, refer to Table A1 for the number of ring galaxies identified in EAGLE at each snapshot. The blue triangles show the number density rate of these galaxies identified (visually) in 162 deep Hubble Space Telescope (HST) Wide Field Planetary Camera 2 archival images and reported in Lavery et al. (2004). The black unfilled circles show the number density rate measured in Elmegreen \& Elmegreen (2006) by visually inspecting the deep archival images of the Galaxy Evolution from Morphology and SEDs survey (GEMS; Rix et al. 2004) and the Great Observatories Origins Deep Survey (GOODS; Giavalisco et al. 2004). The filled square presents a new measurement for the number density of ring galaxies in the redshift range $1.8<z<2.8$ identified visually in the COSMOS field of the FourStar Galaxy Evolution (ZFOURGE) catalogue field images (Straatman et al. 2016), which will be reported in Yuan et al 2018 (in prep.). Galaxy merger rates in the EAGLE simulation, as presented in Lagos et al. (2018), are also presented in this figure for comparison. The major merger rates (stellar mass ratios of $\geq 0.3$ ) are shown as the black dotted line, minor merger rates (stellar mass ratios of $0.1-0.3$ ) as the magenta dash-dotted line and all merger rates (minor+major) as the blue line.

The lower panel of Figure 4 shows the ratio between the collisional ring galaxies number density and the major (blue line) and minor (red dashed line) merger rates. The black dashed line represents equality. This figure shows that collisional ring galaxies are biased tracers of merger rates and that this bias is independent of redshift. Also, the number density of collisional ring galaxies found in EAGLE simulations broadly agrees with the observed number density within the uncertainties. This is a success for the EAGLE project especially because the calibration of the subgrid physics in these simulations did not include galaxy

1 The number density rate is the number of collisional ring galaxies per comoving volume per time interval. Here, we adopt the time interval between each snapshot for our calculations. 


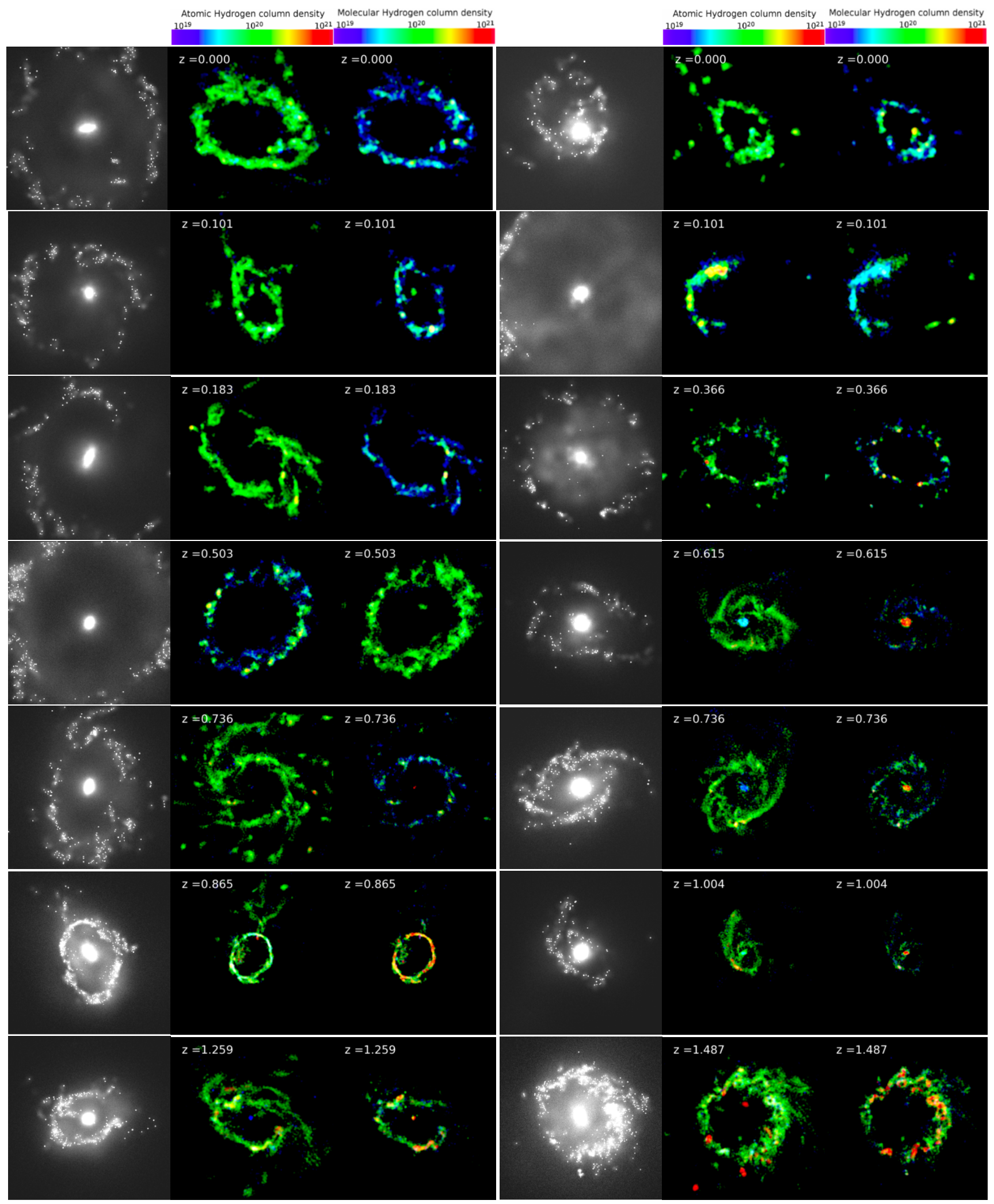

Figure 6. Visualisation of fourteen randomly selected collisional ring galaxies at a redshift range between $z=0$ and 1.5 identified in EAGLE. Each row corresponds to the face-on $u$-band image (left), the $\mathrm{HI}$ (middle) and $\mathrm{H}_{2}$ (left) column density maps of the same galaxy. The colours in the column density images are coded according to the colour bar at the top row and are in units of $\mathrm{cm}^{-2}$. The $u$-band images are $60 \mathrm{kpc}$ on a side while the $\mathrm{HI}$ and $\mathrm{H}_{2}$ column density maps are $100 \mathrm{kpc}$ on a side. 
morphology (Crain et al. 2015). However, it is important to point out the moderate difference in the theoretical prediction of the number density value at $z \sim 0.7$ and 0.9 ; this difference could be due to the various systematic uncertainty sources in the observed number density such as cosmic variance and the uncertainty in the redshift measurements. For instance, the majority of the observed ring galaxies in Lavery et al. (2004) have indirect redshift measurements using a 'standard' absolute $V$-band magnitude for collisional ring galaxies (Appleton \& Marston 1997) to constrain the redshift interval of their sample.

\subsection{The Host Halos of Eagle Ring Galaxies}

The upper panel of Figure 5 shows the mass function for all the haloes at redshift $z=0$ (black line), for haloes hosting a star-forming central galaxy (green dash-dotted line) and for haloes hosting a ring galaxy (red dashed line) at $z=0$. Star-forming central galaxies are those with specific star formation rates $\left(\mathrm{sSFR}=\mathrm{SFR} / \mathrm{M}_{*}\right)>0.01 \mathrm{Gyr}^{-1}$ (Furlong et al. 2015). The lower panel of Figure 5 shows the concentration of haloes (the ratio between the virial radius to the characteristic radius of that halo) as a function of their mass at $z=0$. The grey shaded region is the one sigma deviation from the median value (blue triangles). The red and black symbols show the concentrations of haloes hosting central and non-central ring galaxies, respectively. This figure shows that ring galaxies found in EAGLE live in massive groups that are preferentially more concentrated than other groups that host no ring galaxies at fixed halo mass, in spite of the low number statistics. This is broadly consistent with the observations, which show that ring galaxies are located within galaxy groups that have at least one companion galaxy (Romano et al. 2008). It is reasonable to think that ring galaxies form in dense environments such as compact groups, since a common formation mechanism is based on collisions and drop-through interactions. However, ring galaxies are not found in galaxy clusters, even though the galaxy population density is higher in the cluster environment. The main reason for this is that the high velocity dispersions and/or any off centre encounters between the ring galaxy and other cluster members will disrupt the ring more quickly than in the group environment. This explains the decline in the halo mass function of ring galaxies (red line) with masses larger than $10^{13.3} \mathrm{M}_{\odot}$.

\subsection{The Properties of Eagle Ring Galaxies}

Figure 6 is a visualisation of fourteen randomly selected collisional ring galaxies in EAGLE in the redshift range between $z=0$ and 1.5. Each row corresponds to the face-on $u$-band mock image (left), the $\mathrm{HI}$ (middle) and $\mathrm{H}_{2}$ (left) column density maps of the same galaxy. The $\mathrm{HI}$ and the $\mathrm{H} 2$ column densities were calculated using the prescriptions in Rahmati et al. (2013) for the neutral hydrogen fraction in gas particles and Gnedin \& Kravtsov (2011) for the $\mathrm{HI}$ to $\mathrm{H}_{2}$ transition, as described in detail in Lagos et al. (2015). The colours in the column density images are coded according to the colour bar in the top row and are in units of atoms per $\mathrm{cm}^{2}$. The $u$-band images are $60 \mathrm{kpc}$ on a side while the $\mathrm{HI}$ and $\mathrm{H}_{2}$ column density images are $100 \mathrm{kpc}$ on a side. The atomic and molecular hydrogen column densities exhibit the same ring morphology seen in the $u$-band images, and regions with very low column density "holes" are present in the $\mathrm{HI}$ and the $\mathrm{H}_{2}$ maps

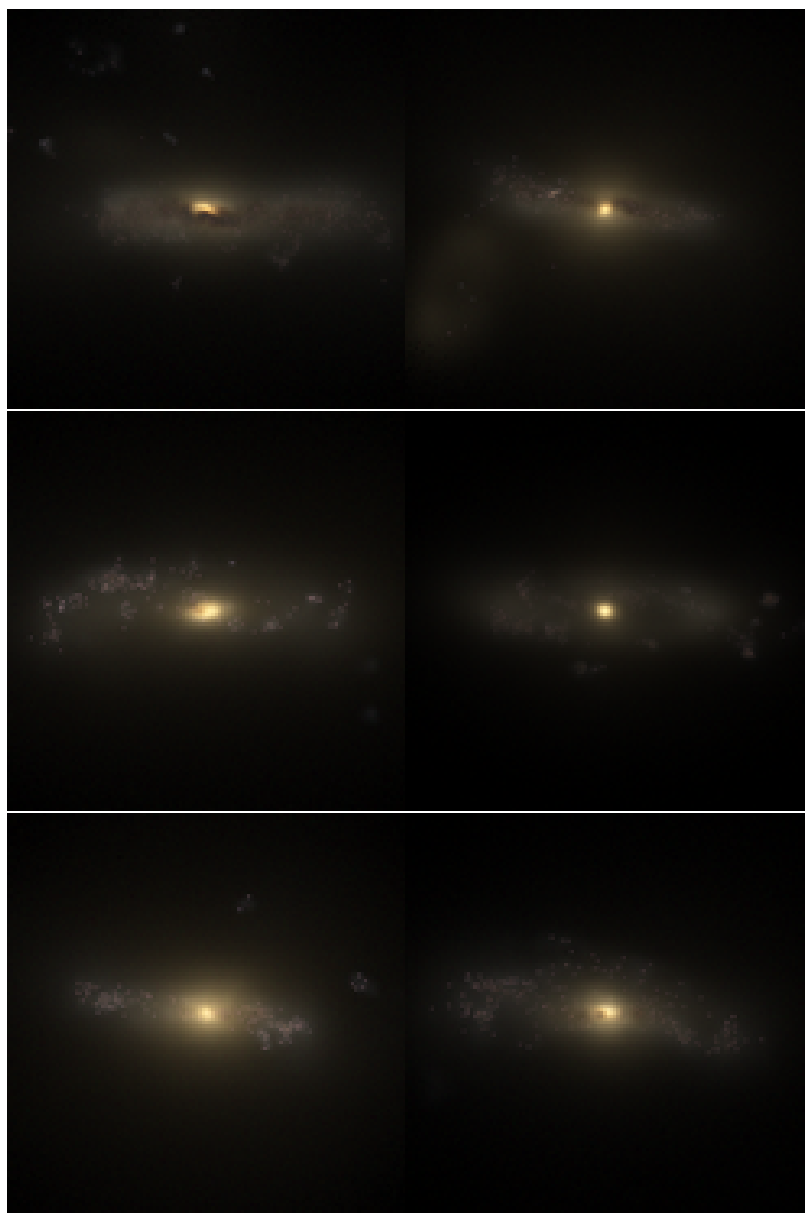

Figure 7. Three-colour gri edge-on images of a subsample of EAGLE ring galaxies identified in the redshift range between $z=0$ and 1 . These images are $60 \mathrm{kpc}$ on a side and are available in the EAGLE database webpage (McAlpine et al. 2016).

around the nucleus and the inner low surface brightness regions. This is in agreement with the current theoretical understanding of the formation history of ring galaxies, in which the collision with a companion galaxy carries the gas of the target galaxy through its disk and forms the ring morphology. The majority of the observed collisional ring galaxies in the local Universe show similar morphologies, for instance the atomic gas in the Cartwheel and AM0644-741 (Lindsay-Shapley Ring) is mostly concentrated in a ring morphology and a region of very low column density is clearly visible in the nucleus; for reference see the HI maps of these two galaxies in Higdon (1996) and Higdon et al. (2012).

Figure 7 presents the edge-on gri colour composite images of a subsample of EAGLE ring galaxies identified between $z=0$ and 1 . The majority of the EAGLE rings have relatively thin stellar disks when viewed edge-on. This is in agreement with noncosmological isolated interaction simulations (Gerber et al. 1996; Mapelli \& Mayer 2012), in which the rings in systems formed due to collisions with massive satellites (mass ratio $\mathrm{M}_{\text {comp }} / \mathrm{M}_{\text {ring }} \geq 1.0$ ) contain larger fractions of their disk's material (gas and stars) and are thicker in comparison with their counterparts which formed with less massive companions. Hence, EAGLE rings are expected to have relatively thin disks as the vast majority of these systems have less massive companions and stellar mass ratios less than one. 


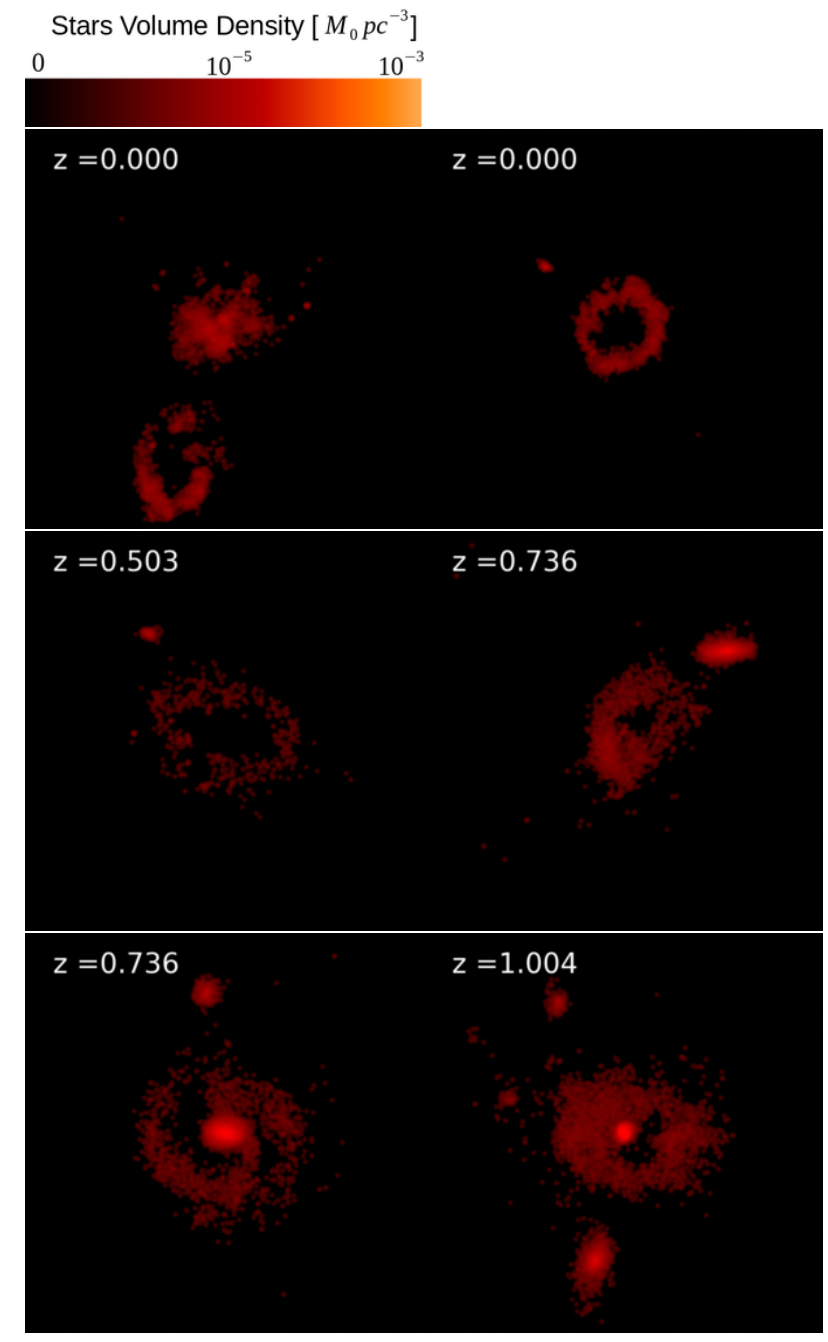

Figure 8. Visualisation of the stellar volume density view in six randomly selected collisional ring galaxies identified in EAGLE between $z=0$ and 1 , as labelled in each panel. In each view, a second galaxy (satellite) appears within a close distance (roughly one ring diameter) from the central ring galaxy. These maps are $200 \mathrm{kpc}$ on a side.

Figure 8 shows the stellar volume density view in six ring galaxies identified in EAGLE between $z=0$ and 1 . The volume density is calculated as the stellar mass in each cell divided by the cubic smoothing length, $\frac{4}{3} \pi h^{3}$, with the image pixels smoothed over $1 \mathrm{kpc}$. In each view, a companion satellite galaxy appears within a close distance (roughly one ring diameter) from the central ring galaxy. We also note that the vast majority of EAGLE ring galaxies have companion satellite(s) that lie within similar distances to the examples in Figure 8, on average within a distance $\gtrsim 100 \mathrm{kpc}$. This agrees with the current theoretical and observational understanding of ring galaxies in the local Universe. For instance, in most of the observed ring galaxies the intruder lies within a physical distance of less than $100 \mathrm{kpc}$ (Elagali et al. 2018; Conn et al. 2016; Fogarty et al. 2011).

Figure 9(a) shows a scatter plot of EAGLE galaxies in the $(u-r)$ colour-stellar mass plane at $z=0$ (left) and $z=0.5$ (right). The colours here are intrinsic, i.e., rest-frame and dust-free colours; refer to Trayford et al. (2015) for details on the dust modelling and the magnitude measurements. The red stars in this
Figure correspond to ring galaxies in EAGLE at $z=0$ (left) and $z=0.5$ (right). The red and blue lines mark the location of the red sequence and blue cloud at each redshift, respectively, and are shown to highlight the colour bimodality of the galaxies in EAGLE (Trayford et al. 2016). Most of the ring galaxies at $z=0$ are located in the blue cloud in the colour-stellar mass diagram which indicates that these systems are actively forming stars. This is comparable to the observations of ring galaxies in which most of the studied collisional ring galaxies (within redshift range $z=0-0.1$ ) are star-forming blue galaxies (Wong et al. 2006; Romano et al. 2008; Fogarty et al. 2011; Parker et al. 2015; Conn et al. 2016; Elagali et al. 2018). However, at $z=0.5$ ring galaxies are located in the green valley with fewer candidates in the blue cloud. This suggests that the interactions driving the ring morphology are also driving the colour transformation and gas exhaustion which may lead to quenching in these galaxies.

Figure 9(b) presents the standard HI gas fraction scaling relation $\left(\mathrm{M}_{\mathrm{HI}} / \mathrm{M}_{*} \mathrm{vs} . \mathrm{M}_{*}\right)$ of the EAGLE galaxies at $z=0$ (left) and $z=0.5$ (right). At both redshifts, the majority of the ring galaxies have higher gas fraction in comparison with the median at fixed stellar mass. This highlights the high HI gas fraction in collisional ring galaxies found in the EAGLE simulations, which agrees well with the observations, in which collisional ring galaxies are known to have high HI gas fractions relative to other galaxies at fixed stellar mass (Elagali et al. 2018). Figure 9(c) shows the SFR vs. the stellar mass of EAGLE galaxies at $z=0$ (left) and $z=0.5$ (right). The black line shows the star formation main sequence at each redshift (Furlong et al. 2015). At redshift $z=0$, the majority of ring galaxies are active star-forming galaxies and lie on the main sequence with few occupying the green valley. On the other hand, at redshift $z=0.5$ ring galaxies have considerably lower star formation rates at fixed stellar mass than the main sequence. This is consistent with the top right panel of the same Figure, where ring galaxies appear to be in the process of quenching and occupy the green valley in the colour-stellar mass plane. It is important to note that massive galaxies in EAGLE $\left(\mathrm{M}_{*} \gtrsim 10^{10} \mathrm{M}_{\odot}\right)$ are known to have slightly less $\mathrm{HI}$ gas than in observations (Crain et al. 2017), which is expected to have a direct impact on the star formation rates resulting from the interaction. This can be part of the reason why ring galaxies in EAGLE have less star formation rate in comparison with the observations, see for example the early IRAS study of ring galaxies in Appleton \& Struck-Marcell (1987). We study the ISM of ring galaxies in the next subsection to explore any other physical reasons that may lead to the low star formation in ring galaxies in EAGLE. Figure 9(d) shows the HI gas fraction $\left(\mathrm{M}_{\mathrm{HI}} / \mathbf{M}_{*}\right)$ versus the specific star formation rate for galaxies in the EAGLE simulation at $z=0$ (left) and $z=0.5$ (right). The black line marks the median values at each SSFR bin. At redshift $z=0.5$, the majority of the ring galaxies have a higher gas fraction than the median value at their SSFR bin. This is different for ring galaxies at redshift $z=0$ as only two rings have higher gas fraction at fixed sSFR.

It is important to mention that we explored these standard scaling relations up to $z=1.5$, however we only show two redshifts for brevity. Ring galaxies at higher redshifts have the same trend as rings at $z=0.5$; the majority of these systems have high gas fractions yet lie below the star formation main sequence and occupy the green valley in the colour-stellar mass plane. Combining the results of the $\mathrm{HI}$ and the SFR of ring galaxies versus the general population of galaxies in EAGLE, we see that 

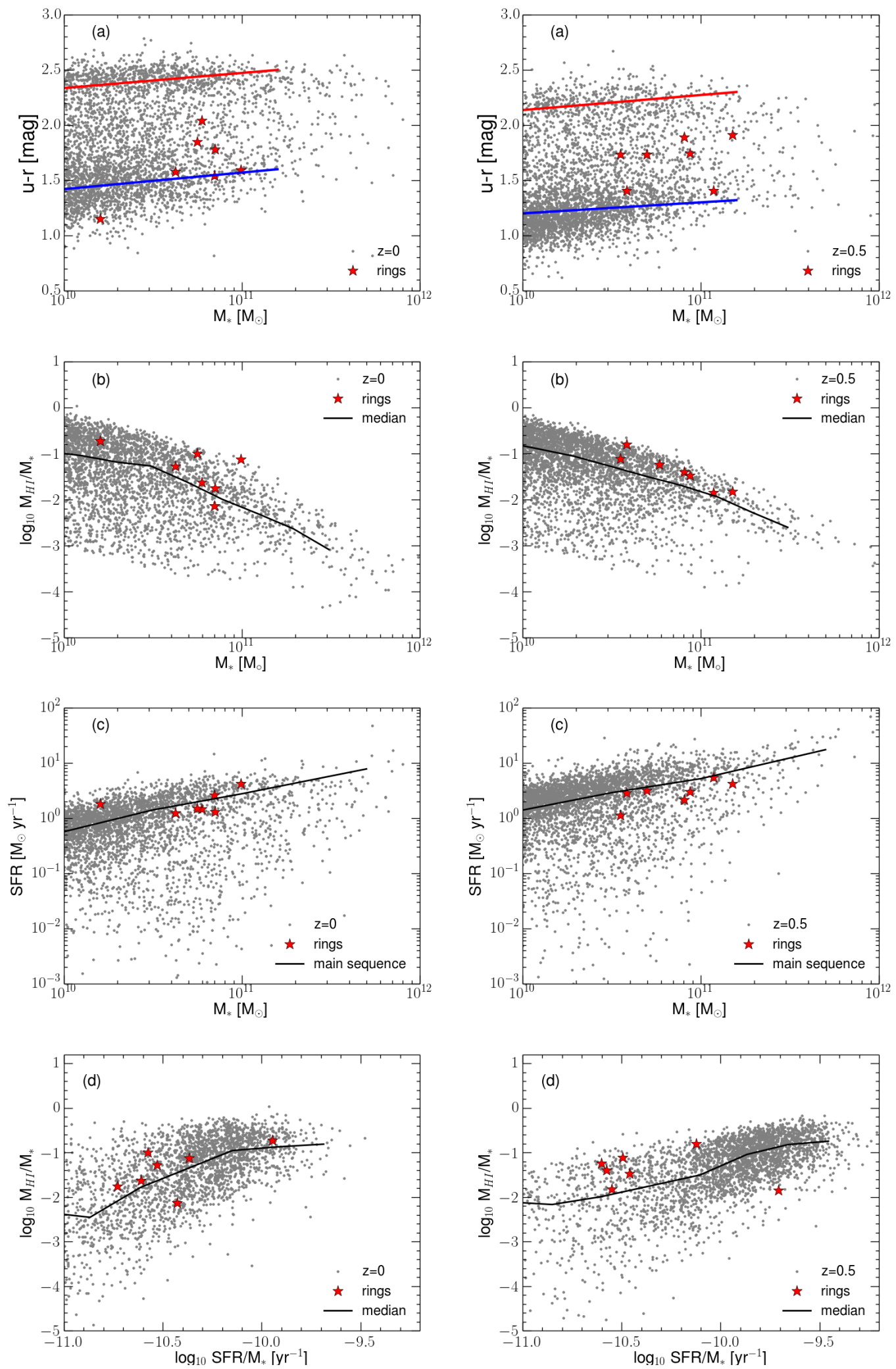

Figure 9. Panels (a) show a scatter plot of EAGLE galaxies in the colour-stellar mass plane $\left(u-r\right.$ vs. $\left.\mathbf{M}_{*}\right)$ at $z=0$ (left) and $z=0.5$ (right). The red and blue lines mark the location of the red sequence and blue cloud at each redshift (Trayford et al. 2016), respectively. Panels (b) present the HI gas fraction scaling relation $\left(\mathrm{M}_{\mathrm{HI}} / \mathrm{M}_{*}\right.$ vs. $\mathrm{M}_{*}$ ) of EAGLE galaxies (grey circles), and the black line marks the median gas fraction values at fixed stellar mass. Panels (c) show the star formation rate (SFR) versus the stellar mass for galaxies in EAGLE at $z=0$ (left) and $z=0.5$ (right). The black line in this panel shows the star formation main sequence at each redshift (Furlong et al. 2015). Panels (d) show the HI gas fraction $\left(\mathrm{M}_{\mathrm{HI}} / \mathrm{M}_{*}\right)$ versus the specific star formation rate $\left(\mathrm{SFR} / \mathrm{M}_{*}\right)$ for galaxies in EAGLE at $z=0$ (left) and $z=0.5$ (right). The red stars in this figure represent the EAGLE ring galaxies at each redshift. 

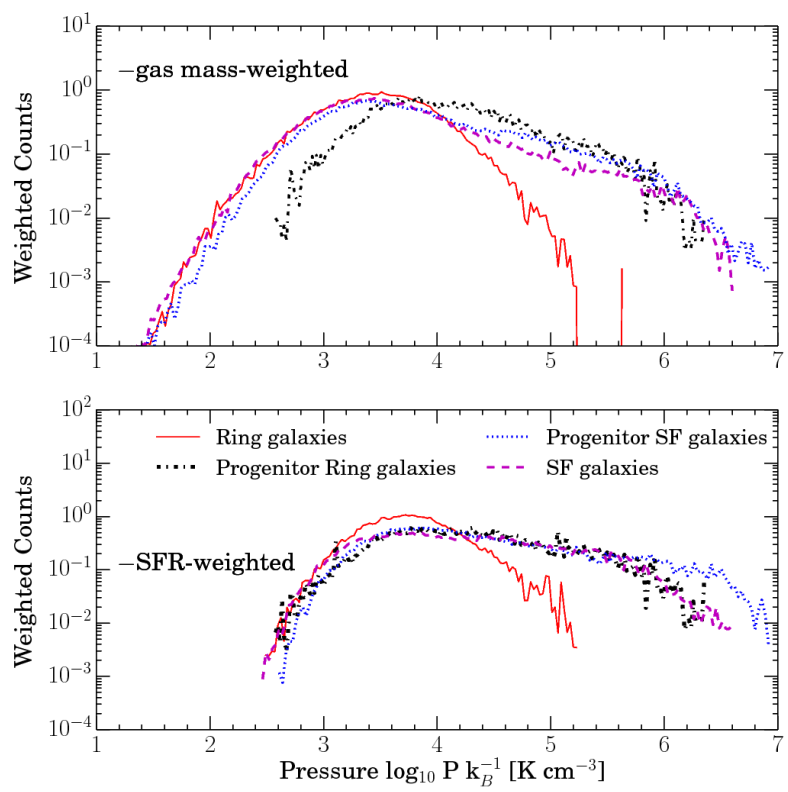

Figure 10. The pressure of the gas particles in the EAGLE ring (red line) and control (magenta dashed line) galaxies at similar redshift expressed in terms of $P k_{\mathrm{B}}^{-1}$, where $\mathrm{k}_{\mathrm{B}}$ is Boltzmann's constant. The black dash-dotted and blue dotted lines show the pressure PDF of the ring and control galaxies two snapshots before ( $z=0.7$ ), respectively. In the upper panel the pressure of the gas particles is normalised and weighted by their neutral gas mass, whereas the lower panel presents the pressure normalised and weighted by their SFR
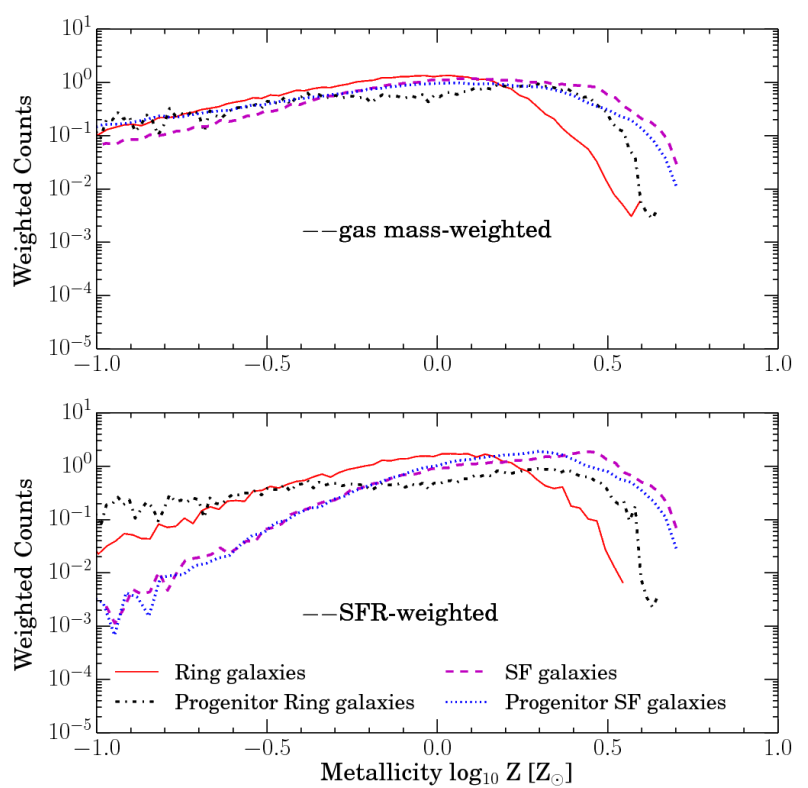

Figure 11. The metallicity of the gas particles in the EAGLE ring (red line) and control (magenta dashed line) galaxies expressed in units of solar metallicities. The black dash-dotted and blue dotted lines show the metallicity PDF of the progenitor ring and control galaxy samples at $z=0.7$, respectively. In the upper panel the metallicity of the gas particles is normalised and weighted by their neutral gas mass, whereas the lower panel presents the metallicity normalised and weighted by their SFR. ring galaxies are characterised by an inefficient conversion of the $\mathrm{HI}$ gas into stars. It is important to caution the readers to the low number statistics in this analysis; even though it is evident that rings at higher redshifts $(z \gtrsim 0.5)$ exhibit inefficiency in star formation these systems maybe unrepresentative of the whole population of ring galaxies at higher redshifts. We discuss the physical drivers behind this inefficiency in Section 4.4.

\subsection{The ISM of Eagle Ring Galaxies}

EAGLE adopts the star formation law of Schaye \& Dalla Vecchia (2008), which expresses the Kennicutt (1998) observational relation as a pressure law, with the SFR of an individual gas particle scaling as $\mathrm{P}^{n-1 / 2}$, with $\mathrm{n}=1.4$. The gas particles, however, are only assigned a SFR if they reach a certain density level, which depends on the metallicity of the gas (Schaye 2004). This means that lower pressure directly translates into a lower SFR. To better understand the ISM of ring galaxies and the reasons behind the high amount of $\mathrm{HI}$ gas and the inefficient star formation in these system, we explore the properties of the gas particles in seven ring galaxies at redshift $z=0.5$ and compare them with seven other EAGLE star-forming galaxies with similar gas and stellar masses. We refer to the latter as the control galaxy sample. The average difference in the $\mathrm{HI}$ gas mass between the ring and control samples is smaller than 0.05 dex.

Figure 10 shows the pressure of the gas particles in ring (red line) and control (magenta dashed line) galaxies at the same redshift expressed in terms of $P k_{\mathrm{B}}^{-1}$, where $\mathrm{k}_{\mathrm{B}}$ is Boltzmann's constant. The black dash-dotted and blue dotted lines show the pressure profile of the ring and control galaxies two snapshots before the ring feature is identified $(z=0.7)$, respectively. We refer to those as the progenitors of ring and control galaxies. Here, we adopt a $50 \mathrm{kpc}$ spherical aperture centred on the subhalo's centre of mass and consider all the material within this aperture to belong to the subhalo (the galaxy). The upper panel of Figure 10 shows the pressure of the gas particles weighted by their neutral gas masses, whereas the lower panel presents the same property weighted by SFR.

There is a clear difference in the gas pressure between the ring and the control (star-forming) galaxy samples. The gas in the progenitor of both the ring and control galaxies, as well as and control galaxies at $z=0.5$ have higher pressure regardless of the weighting. This is one reason for the inefficient star formation processes taking place in ring galaxies and can partly explain the excess amount of HI gas in these system. This is because the gas-phase pressure affects the rate at which the HI gas is converted to molecular hydrogen, which physically is expected to regulate the conversion into stars (Elmegreen 1989; Elmegreen \& Parravano 1994; Elmegreen 1993; Blitz \& Rosolowsky 2006; Leroy et al. 2008), and in some models is the only parameter controlling whether molecular or atomic gas dominates the ISM in galaxies (Lagos et al. 2011). This means that high column density, low-pressure $\mathrm{HI}$ gas has relatively few $\mathrm{H}_{2}$ molecules and therefore a low SFR in comparison with high column density, high-pressure $\mathrm{HI}$ gas. We note that the gas-phase pressure of the particles evolves with redshift, see Figure 12 in Lagos et al. (2015), which is the reason for the shift in the pressure profile of the two progenitor samples at $z=0.7$ towards higher values overall compared to the ring and control galaxy samples at $z=0.5$, especially in the upper 

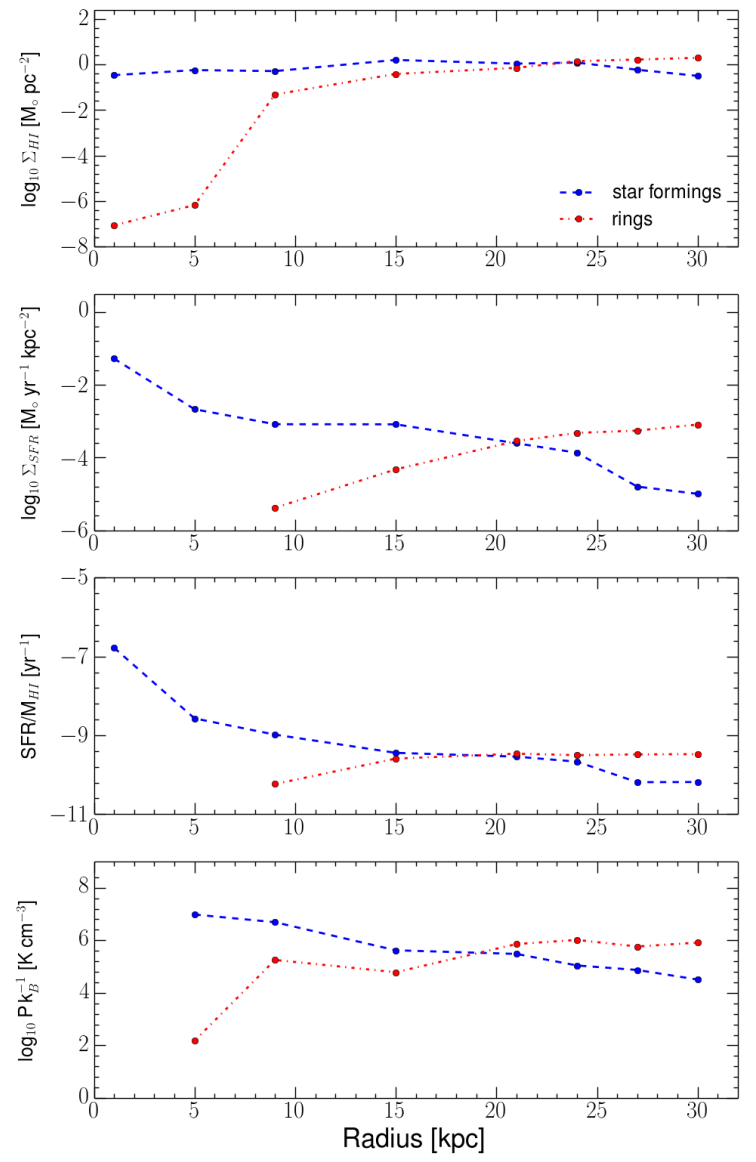

Figure 12. The median radial profile of the HI gas surface density $\Sigma_{\mathrm{HI}}$ (top panel), the star formation rate surface density $\Sigma_{\mathrm{SFR}}$ (second panel), the specific star formation rate SFR/M $\mathrm{M}_{\mathrm{HI}}$ (third panel), and the hydrostatic pressure of the gas (bottom panel) in the control seven star-forming galaxies (blue) and EAGLE ring galaxies (red) at $z=0.5$.

panel.

Figure 11 shows the metallicity of the gas particles in the previously discussed seven rings (red line) and control (magenta dashed line) galaxy samples expressed in units of solar metallicities. The black dash-dotted and blue dotted lines present the metallicity profile of the progenitors of the ring and control galaxies at $z=0.7$, respectively. The upper panel of Figure 11 shows the metallicity of the gas particles weighted by their neutral gas masses, whereas the lower panel presents the metallicity weighted by their SFR. It is evident from Figure 11 that the gas particles in ring galaxies have lower metallicities compared to the control galaxy sample. It is important to note that the difference in the metallicity profile between the ring galaxies and their progenitors is likely due to the interaction with the dwarf satellite in which the ring galaxies may gain low metallicity gas from the dwarf as a result of the interaction.

An intriguing question to address is whether the overall low gas-phase pressure in ring galaxies is due to the different gas distribution in these systems, in which most of the gas piles up in regions of low pressure, or whether the gas-phase pressure in these galaxies is low irrespective of radii. In the latter case, the pressure at a given radius would be lower than that of the control sample. We explore these two scenarios in Figure 12. This figure presents the median radial profile of the HI gas surface density $\Sigma_{\mathrm{HI}}$ (top panel), the star formation rate surface density $\Sigma_{\text {SFR }}$ (second panel), the specific star formation rate SFR/M $\mathrm{M}_{\mathrm{HI}}$ (third panel), and the hydrostatic pressure of the gas (bottom panel) in the control seven star-forming galaxies (blue) and the ring galaxies (red) at $z=0.5$. It is important to note that these ring galaxies have different physical disk radii, hence their ring features are not necessarily at the same radius. The two different samples have similar neutral hydrogen gas surface densities at radii $r>5 \mathrm{kpc}$ but differ dramatically at radii $r \lesssim 5 \mathrm{kpc}$. This is connected to the suppression of the star formation and pressure in the inner regions of ring galaxies when compared to the control sample. However, this suppression takes place out to larger radii than the depression in the HI surface density of ring galaxies. These trends show that both scenarios described above are taking place in our ring galaxies in EAGLE. At radii $r>20 \mathrm{kpc}$, ring galaxies have higher hydrostatic pressure in comparison with their counterparts. The drop in the gas-phase pressure seen in the inner regions of ring galaxies is caused by the drop-through collision, which leads to a "dilution" in the ISM gas surface density and as a consequence the star formation surface density drops significantly in the inner radii of these galaxies. This is evidenced by the progenitors of ring galaxies having the same pressure distributions as the progenitors of the control sample.

The relatively low pressure and metallicity in ring galaxies offers a physical interpretation for the inefficient HI to SFR conversion, mostly in the inner regions of these systems, which can lead to the high $\mathrm{HI}$ gas fraction found in observations of ring galaxies (Elagali et al. 2018). Further, this can also explain the low total molecular hydrogen masses in some of the observed ring galaxies in the local Universe. For instance, the Lindsay-Shapley ring galaxy has as much total $\mathrm{H}_{2}$ mass as a typical dwarf galaxy (Leroy et al. 2005; Higdon \& Higdon 2010), which is surprising especially because this galaxy has an atomic gas mass of $\mathrm{M}_{\mathrm{HI}}=3 \times 10^{10} \mathrm{M}_{\odot}$ (Higdon et al. 2012). Recent observational studies of ring galaxies show that some of these galaxies are $\mathrm{H}_{2}$ deficient even in the outer ring where the atomic hydrogen surface density is highest. The most plausible scenario offered in these studies is that the molecular hydrogen in the ring is destroyed by ultraviolet photons from $\mathrm{OB}$ stars born in the ring's confined environment (Higdon et al. 2015; Wong et al. 2017). This is different than the result presented in Figure 12, where the star formation rate surface density in ring galaxies is higher in the outer radii $(r>20 \mathrm{kpc})$ in comparison with the star-forming galaxy sample implying that the abundance of cold gas in the ring should be relatively normal. To examine the photodissociation hypothesis suggested in Higdon et al. (2015); Wong et al. (2017), we need to follow the formation and the evolution of the molecular hydrogen. This requires a detailed description of the ISM and the photoionisation effects of local sources on the $\mathrm{H}_{2}$ gas, which is not currently feasible in simulations with a boxsize as large as EAGLE and only prescribed in zoom-in simulations, see for example Hopkins et al. (2014, 2017). However, future developments in large hydrodynamical simulations, such as EAGLE, will explicitly track the formation of the molecular hydrogen at much higher resolution. Such projects 

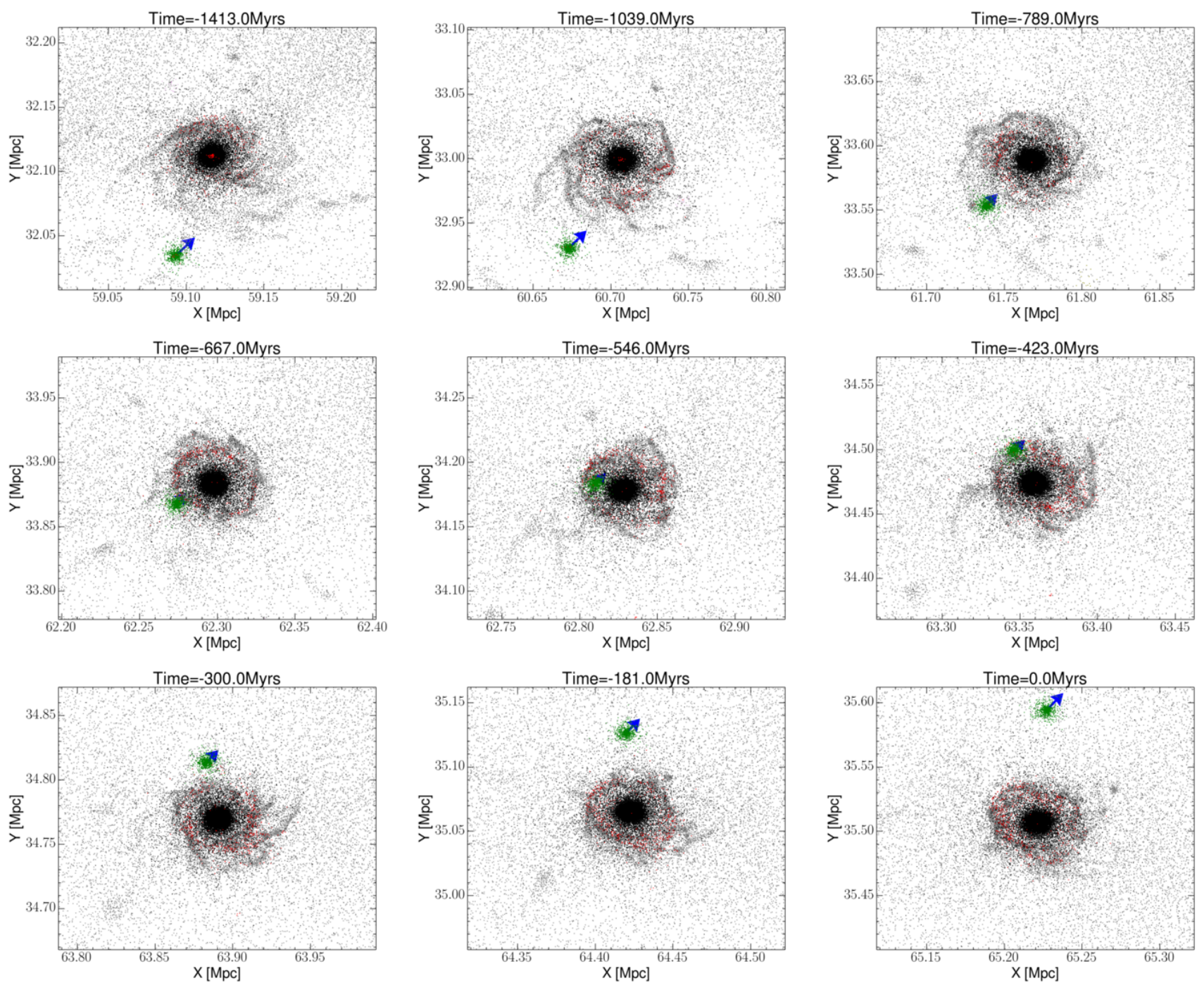

Figure 13. The morphological evolution of a gas-rich spiral in EAGLE colliding once with a dwarf galaxy. In all the maps the system is viewed down the simulation z-axis. The grey dots show the gas particles, while the red and black dots show newly formed stars (age $<30 \mathrm{Myr}$ ) and those with age $>30 \mathrm{Myr}$, respectively. The green dots show the stellar particles of the dwarf companion. This system is identified as a ring galaxy at $z=0$. The time of the snipshot in Megayears with respect to the ring formation time $(z=0)$ is denoted at the top of each panel. The blue arrows show the magnitude and the direction of the velocity vector of the dwarf galaxy. We print half the number of particles to avoid overcrowding.

will be ideal to address this issue.

\section{FORMATION MECHANISMS OF EAGLE RING GALAXIES}

To understand the formation mechanisms of ring galaxies in EAGLE, we trace the evolution of the haloes hosting ring galaxies using the finer time resolution snipshots (Schaye et al. 2015). The time resolution of these snipshots span between $0.05-0.3 \mathrm{Gyr}$, which is smaller than the typical merger timescale (Ji et al. 2014; Jiang et al. 2008), hence very suitable to track collisions and drop-through interactions. For each ring galaxy, we examine the history of their host halo by tracing its evolution for one Gigayear before and one Gigayear after the ring morphology is identified.

We visually inspect the history of the all the ring galaxies
Table 2. The formation mechansims of ring galaxies in the EAGLE simulations.

\begin{tabular}{lll}
\hline \hline Mechanism & Number & Averaged lifespan (Myr) \\
\hline One collision encounter & 37 & 700 \\
Multiple collision encounters & 9 & 1100 \\
Barred galaxies (P-type) & 9 & 2400 \\
\hline
\end{tabular}

identified in EAGLE to determine whether the ring morphology forms in response to collisions or not. This process is very simple, as in all the cases the effect of the drop-through is evident if present. An example of this is shown in Figures 13-14 (discussed in detail below). The majority of ring galaxies identified in EAGLE (46 out of 55 galaxies) have an interaction origin, i.e., formed when a companion galaxy(ies) drop-through a "target" galaxy (sometimes more than one drop-through). The remainder galaxies (17 per cent) have very long-lived ring morphologies ( $>2 \mathrm{Gyr}$ ). These long-lived systems are most likely barred galaxies (P-type 

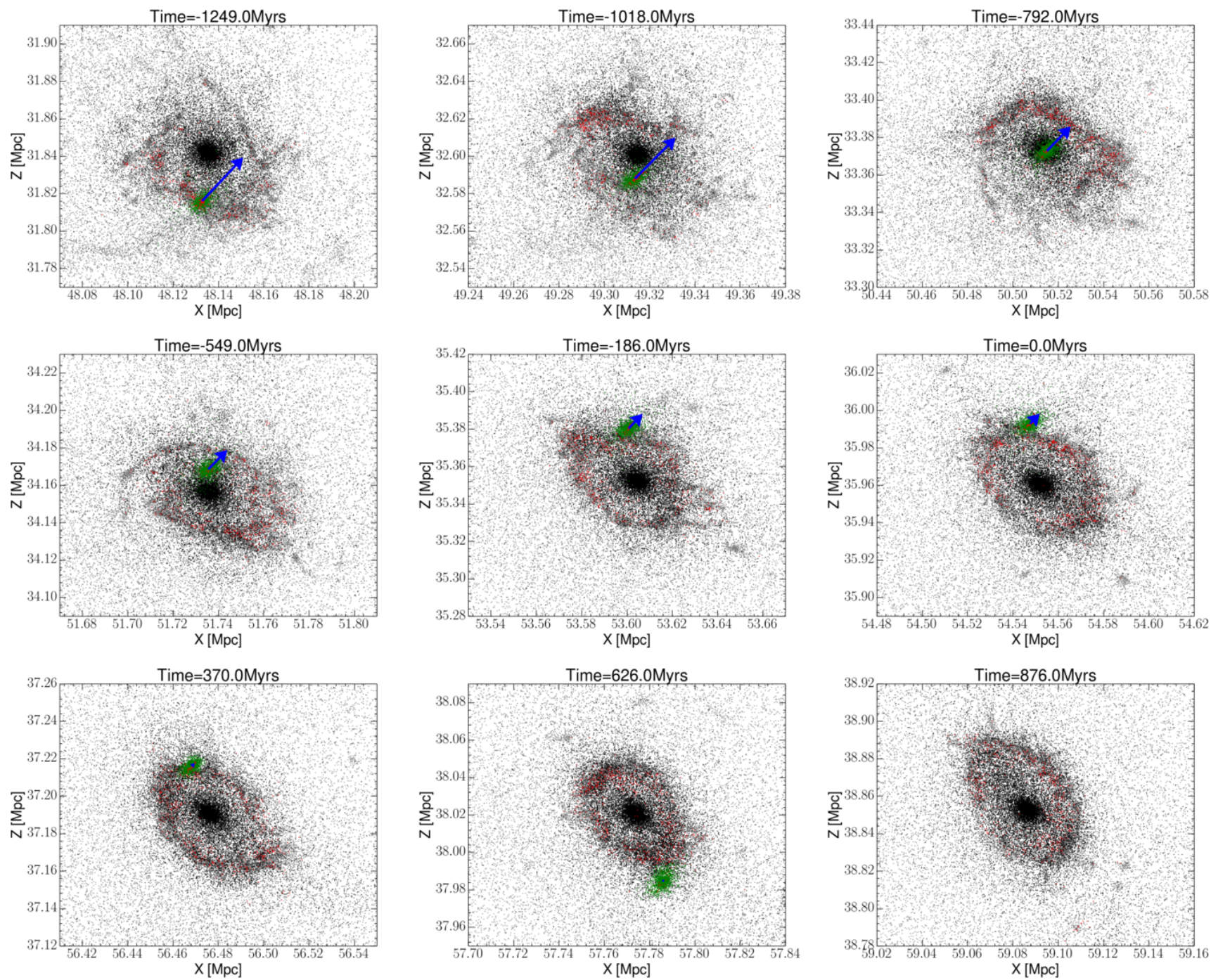

Figure 14. The morphological evolution of a gas-rich spiral in EAGLE colliding twice with a dwarf galaxy. In all the maps the system is viewed down the simulation y-axis. The gas and stars are colour-coded similar to Figure 13. This system is identified as a ring galaxy at $z=0.5$. The time of the snipshot in Megayears with respect to the ring formation time $(z=0.5)$ is denoted at the top of each panel. The blue arrows show the magnitude and the direction of the velocity vector of the dwarf galaxy. We print half the number of particles to avoid overcrowding.

rings). For instance, one of these long-lived ring galaxy hosts, identified at $z=0$, is listed as a strongly barred galaxy in A17. Table 2 lists the different identified formation mechanisms for ring galaxies in EAGLE and the average lifespan of the ring morphology in each scenario. Figure 13 presents the interaction history of a gas-rich, spiral colliding with a dwarf companion galaxy at redshift $z=0$. The ring morphology in this scenario is induced due to a single off-centre collisional encounter with a dwarf companion. As listed in Table 2, the majority of ring galaxies in EAGLE are formed in this scenario. Figure 14 presents the second formation scenario for ring galaxies in EAGLE at redshift $z=0.5$. In this case, a gas-rich, bulgeless spiral galaxy collides with a dwarf galaxy multiple times, i.e., more than one drop through collision. This second scenario is expected since the intruder and the target galaxy are more bound after the first encounter as a result of the dynamical friction. In EAGLE, this scenario often prompt a full merger after a few hundred megayears from the second encounter. Multiple encounters can prolong the lifespan of the ring and results in different kinematics for the gas and stars of target galaxy (see
Figure 19), the lifespan of the ring morphology for these scenarios is shown in Figure 17 (discussed in detail below). Figure 15 shows a subsample of the P-type (long-lived) ring galaxies identified in EAGLE at $z \leq 1.5$. The ring morphology in this class is mainly due to the strong bars present in these galaxies. These systems have ring features that last for $>2 \mathrm{Gyr}$ and host strong bars that are evident in the gri mock images of this figure.

Figure 16 shows the stellar mass ratio between the companion and the ring galaxy $\left(\mathrm{M}_{\text {comp }} / \mathrm{M}_{\text {ring }}\right)$ in the 84 per cent of our sample of ring galaxies, in which we find a clear connection between the ring morphology and galaxy interactions. The median stellar mass ratio in this sample equals 0.14 , which is in agreement with the observational and theoretical studies of these systems. The stellar mass ratios in most of the observationally studied ring galaxy pairs lie between 0.1 to 1 (see for example Higdon \& Wallin 1997; Higdon 1995; Wong et al. 2006; Romano et al. 2008; Parker et al. 2015; Wong et al. 2017). Only a handful of ring galaxy pairs have ratios outside this range. For instance, three known ring galaxies 

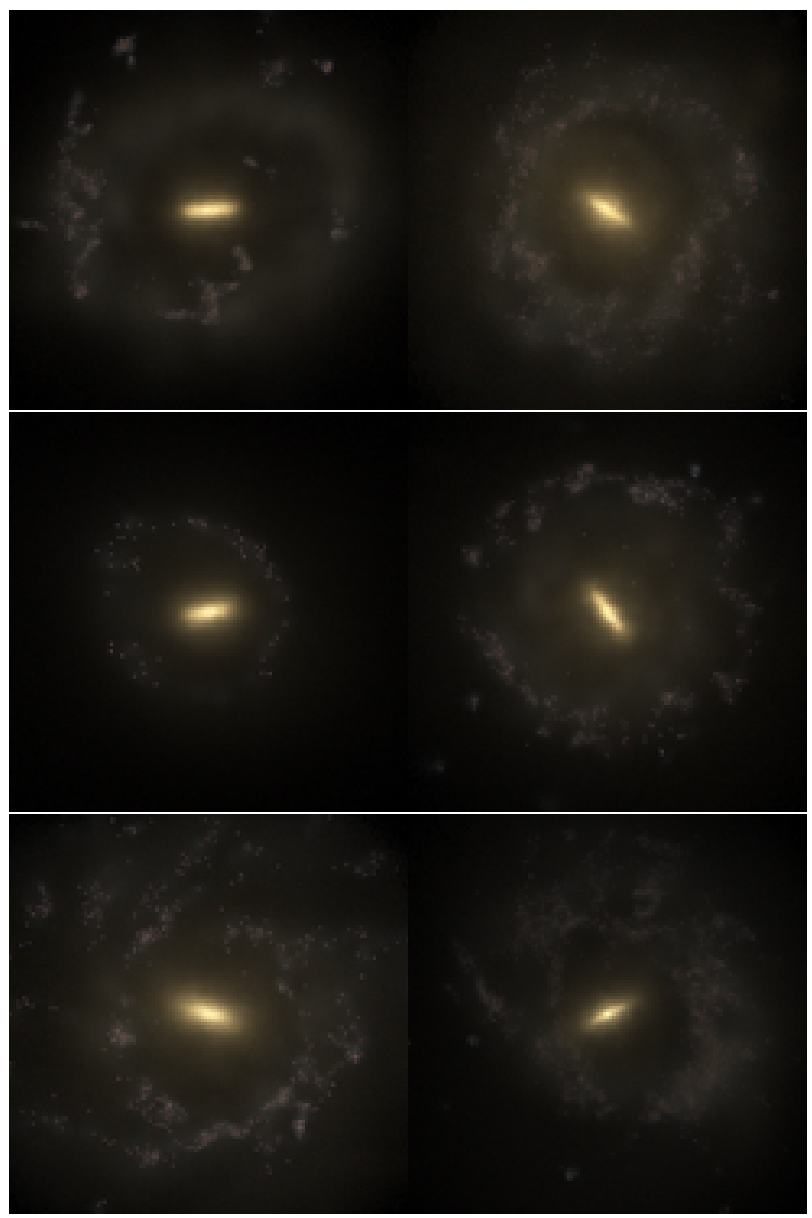

Figure 15. Three-colour gri mock images of a subsample of the long-lived EAGLE ring galaxies identified in the redshift range between $z=0$ and 1.0. These images are $60 \mathrm{kpc}$ on a side and are available at the EAGLE database webpage (McAlpine et al. 2016).

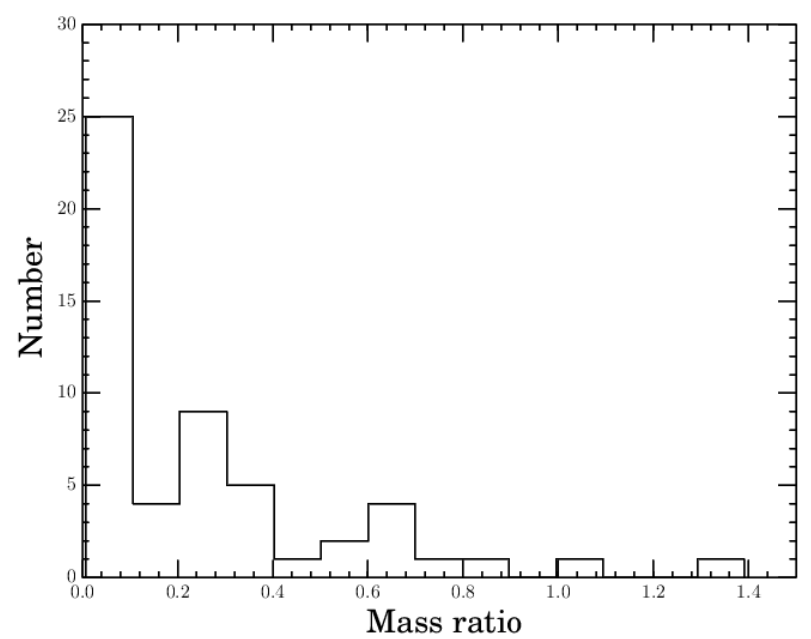

Figure 16. The stellar mass ratio between the companion and the ring galaxies for our sample of rings in EAGLE that formed due to collisions. Here, we show all rings at $z \leq 1.5$.

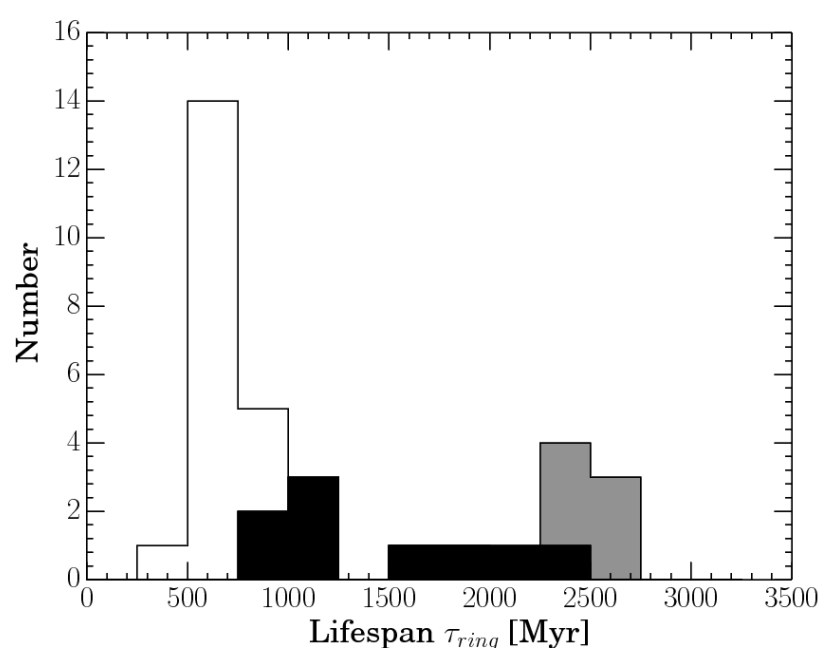

Figure 17. The lifetime of the ring of star formation morphology in our EAGLE ring galaxies at $z \leq 1.5$. The unfilled histogram shows the lifespan of the ring galaxies formed with one companion dropping once through its gas disk, while the black filled histogram show those which have companion(s) plunging through more than once. The grey histogram represents the long-lived ring galaxies, those galaxies have very strong bars.

have mass ratios smaller than 0.1 , namely IZw45 (ratio 0.04), NGC 2793 (ratio 0.05) and NGC 922 (ratio 0.06), while three others have mass ratios larger than one in which the companion is twice the size of the ring galaxy, namely, the Arp 141, Arp 147 and Arp 148 (Romano et al. 2008). In EAGLE, we identify two ring galaxies that have more massive companions. The interaction in these two cases is not a "bullseye" collision but rather a collision with large impact parameter, i.e. similar to a flyby interaction.

Figure 17 also shows the lifetime of the star-forming ring morphology found in our ring galaxy sample. The lifetime of the ring is determined from when the collision occurs between the target and companion galaxies in the case of collision induced rings, i.e., from the time when the companion is closest to the target until the time when the ring structure starts to collapse and hosts less than 50 per cent of the new stars $(<30 \mathrm{Myr})$ formed in the whole galaxy. The unfilled histogram shows the ring structure lifespan of the ring galaxies formed with one companion dropping once through its gas disk, while the black filled is for those which have multiple companions $(>1)$ plunging through more than once (predominantly identified at higher redshifts). For the latter, the lifetime is measured from the first collision. The grey histogram presents the long-lived ring galaxies found in EAGLE. The median lifespan of the ring morphology in systems formed with one companion dropping once through its disk is $700 \mathrm{Myr}$. Multiple interactions, i.e. more than one companion passing through the disk of the main galaxy or the same companion plunging more than once, significantly prolong the lifespan of the ring feature in the main galaxy.

This value is in broad agreement with the ages of the ring morphology in non-cosmological isolated interaction simulations, which in most cases range between $500 \mathrm{Myr}$ and $1000 \mathrm{Myr}$ (Hernquist \& Weil 1993; Mihos \& Hernquist 1994; Horellou \& Combes 2001; Mapelli et al. 2008b). Figure 18 shows the evolution of the median stellar mass ratio of the interacting galaxy pairs (blue) and the median lifetime of the ring morphology (red) with 


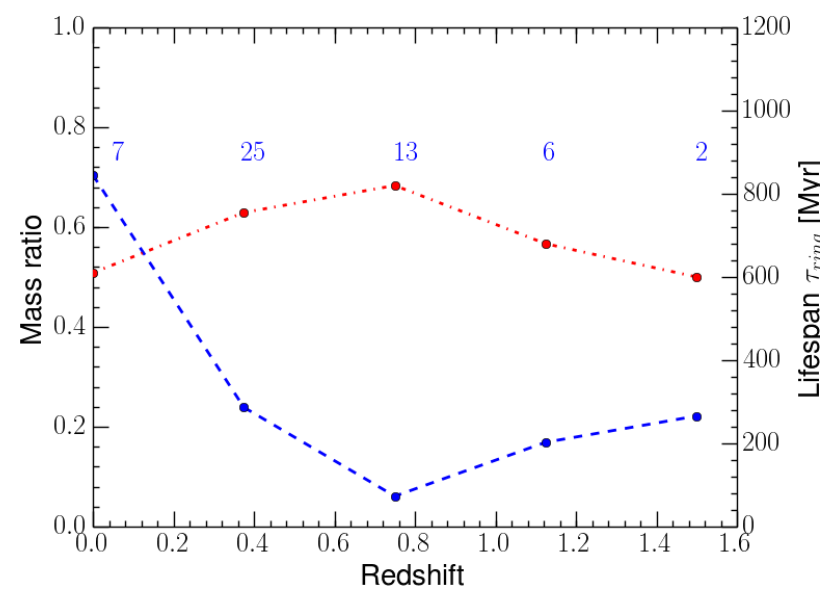

Figure 18. The evolution of the median stellar mass ratio of the interacting galaxy pairs (blue) and the median lifetime of the ring morphology (red). Here, we only show rings that have an interaction origin and exclude the long-lived barred ring systems. Numbers represent the total number of ring galaxies within each redshift bin.

redshift. Here, we only show rings that have an interaction origin and exclude the long-lived barred ring systems. The numbers in this Figure represent the total number of ring galaxies within each bin. The stellar mass ratio of the interacting pairs are the highest at $z=0$, and decrease rapidly with redshift. On the other hand, the lifetime of the ring morphology increases monotonically with redshift until it reaches the maximum at $z=0.75$, with a median age of $\sim 800 \mathrm{Myr}$, and then decreases at higher redshifts. The evolution of the mass ratio is expected as galaxies tend to be more gas rich and smaller at higher redshifts, and consequently the life time of the ring morphology will also evolve. However, the steep drop in the mass ratio can also result from small number statistics. The next generation of hydrodynamical simulations will offer a chance to revisit this with better statistics, as it will simulate galaxies in larger volumes, allowing the exploration of redshift trends more robustly.

Figure 19 presents the radial velocity of the gas particles with respect to the plane of the disk for two ring galaxies in EAGLE at redshift $z=0.5$. The plane of the disk is determined as that perpendicular to the total stellar spin vector of the galaxy. We only consider the component of the gas particles in the radial direction of the plane of the disk. Thus, the radial velocity is a measure of the expansion velocity of the ring. Figure 19a shows the radial velocity of a ring that formed due to one drop-through interaction with a dwarf satellite and is measured at time $t \sim 200 \mathrm{Myr}$ after the encounter. The radial velocity of the ring at $r \gtrsim 17 \mathrm{kpc}$ is positive and indicates expansion, while in the inner regions the velocity is negative which means that the gas is falling back to the centre. For instance, the outer rings have a maximum radial velocity of $v_{\mathrm{rad}}=120 \mathrm{~km} \mathrm{~s}^{-1}$ at a radius of $r=25 \mathrm{kpc}$, whereas at a radius of $r=7 \mathrm{kpc}$ the radial velocity equals $v_{\text {rad }} \sim-160 \mathrm{~km} \mathrm{~s}^{-1}$. This result is in agreement with the predictions of the analytic caustic theory (Appleton \& Struck-Marcell 1996; Struck-Marcell \& Lotan 1990; Struck 2010; Mapelli \& Mayer 2012) and the observations of ring galaxies in the local Universe (Conn et al. 2016; Fogarty et al. 2011; Higdon 1996). Figure 19b shows the radial velocity profile of a long lived ring galaxy formed due to two encounters with a dwarf companion and is measured at time $t \sim 140 \mathrm{Myr}$ after the second encounter. The radial velocity profile in this case is different than in Figure 19a in that the inward radial velocity (infall) in the inner regions of the ring are much smaller in this case and only particles at radii $r \gtrsim 5 \mathrm{kpc}$ have negative velocities. Assuming that the second collision is also impulsive, we expect the velocity of the gas particles to undergo a dramatic change, in which the velocity of the second impulse will be added to the gas particles' current velocity. This means that the particles moving inward (negative infall velocity) will gain momentum which will reduce the net inward negative velocity or even cancel it, while the gas particles moving outward (expanding) will gain more speed and will increase their expansion velocity (refer to Appleton \& Struck-Marcell (1996); StruckMarcell \& Lotan (1990) for more detailed analysis on multiple encounters).

\section{DISCUSSION AND CONCLUSIONS}

In this paper, we use the EAGLE hydrodynamical simulations to study the formation and characteristics of ring galaxies. EAGLE's volume, $(100)^{3} \mathrm{cMpc}^{3}$, allows us to identify a relatively large sample of ring galaxies and quantify their formation mechanisms and evolution with redshift. We characterise our ring galaxy sample, putting special emphasis on their star formation rates (SFRs), colours, metallicities, and atomic (HI) hydrogen gas and how these properties scale with each other. We also characterise their environments. Our main findings are summarised as follows:

- The number density evolution of ring galaxies in the EAGLE simulation is in broad agreement with the observations of Lavery et al. (2004); Elmegreen \& Elmegreen (2006). This means that the numerical treatment of the ISM, star formation, and feedback model in the EAGLE simulation are adequate enough to reproduce a realistic ring-morphology population. This is an unprecedented success for hydrodynamical simulations especially because the calibration of the subgrid physics in EAGLE does not include galaxy morphology (Crain et al. 2015). This is particularly important as other simulations produce an overabundance of ring galaxies (e.g., the Illustris simulations Snyder et al. 2015).

- Ring galaxies live in massive groups $\left(\mathrm{M}_{\text {halo }} \sim 10^{13} \mathrm{M}_{\odot}\right)$ that are preferentially more concentrated than groups without ring galaxies at fixed halo mass. This is in agreement with the observations, in which ring galaxies are located within galaxy groups and have at least one companion galaxy (Higdon \& Wallin 1997; Higdon 1995; Romano et al. 2008; Conn et al. 2016; Wong et al. 2017; Elagali et al. 2018).

- In EAGLE, ring galaxies are moderately star-forming galaxies that typically reside in the green valley, but have high $\mathrm{HI}$ gas fractions. We find that this is due to ring galaxies having an ISM with much lower gas phase-pressure and metallicity than star-forming galaxies with the same stellar and gas masses. By studying the progenitors of ring galaxies, we find that the drop-through collision with the companion(s) is responsible for diluting the gas metallicity and pressure. The latter happens as the gas flows efficiently towards the outskirts of galaxies to form the ring structure, where the pressure is lower.

- The vast majority of ring galaxies identified in EAGLE (83 per cent) have an interaction origin, i.e., are formed when a companion galaxy(ies) drop-through a "target" galaxy (sometimes more than 

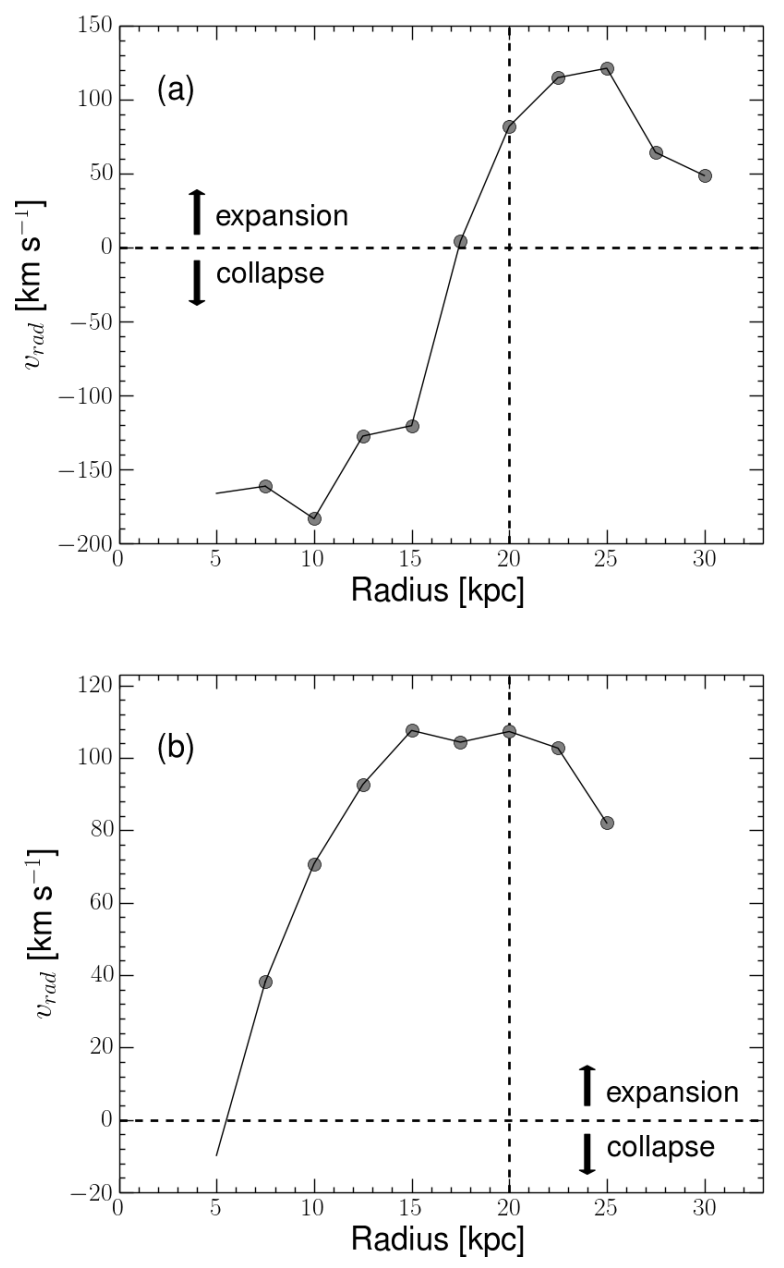

Figure 19. The radial velocity of the gas particles in the plane of the disk for two different EAGLE ring galaxies at redshift $z=0.5$. (a) a ring galaxy formed due to one drop-through interaction with a dwarf satellite. (b) a ring galaxy formed due to multiple drop-through interactions with a dwarf companion (two encounters). The radial velocity is measured after $t \sim 200 \mathrm{Myr}$ from the first encounter in (a) and after $t \sim 140 \mathrm{Myr}$ from the second encounter in (b). The dashed vertical line denotes the approximate location of the ring.

one drop-through). The lifespan of the ring morphology in systems formed with one companion dropping once through its disk is $\sim 700 \mathrm{Myr}$, which is is in broad agreement with the ages of the ring morphology in non-cosmological isolated interaction simulations (Hernquist \& Weil 1993; Mihos \& Hernquist 1994; Horellou \& Combes 2001; Mapelli et al. 2008b). Some ring galaxies form through the effect of more than one companion passing through its disk or the same companion passing more than once. In these cases, we find that the lifespan of the ring feature increases significantly, by a factor of $\sim 2$. We also study the kinematics of EAGLE collisional ring galaxies and find similar results to those predicted by the analytic caustic theory (see e.g., Appleton \& Struck-Marcell 1996; Struck-Marcell \& Lotan 1990). The remainder galaxies (17 per cent) have very long-lived ring morphology ( $>2 \mathrm{Gyr})$, and correspond to barred galaxies (P-type rings).

One aspect of these systems remains unclear mainly due to the limitation of the current hydrodynamical simulations. Some recent observations suggest that ring galaxies are $\mathrm{H}_{2}$ deficient even in the ring, where the HI gas surface density is highest, and hypothesise that in the ring's confined high density ISM, molecular hydrogen is formed due to the high HI surface density but equally destroyed by the continuous UV-field from supernovae and OB stars (Higdon et al. 2015; Wong et al. 2017). To explore the photodissociation effect in the ring's molecular hydrogen, we need to consistently follow the formation and destruction of the molecular hydrogen, which requires a detailed description of the cold phase of the ISM. This is currently not available for large hydrodynamical simulations such as EAGLE, but in smaller box zoom-in simulations is possible and has been implemented to some extent (Hopkins et al. 2014, 2017).

Another limitation of our current analysis is the small number statistics. Ring galaxies are rare systems, as a result we typically find less than ten rings in the entire simulated volume at a given redshift. Thus, any redshift evolution found here is tentative and requires larger volumes, simulated at the same resolution to EAGLE, to confirm them. Using the next generation of hydrodynamical simulations, we will be able to probe larger volumes at the same resolution as the current EAGLE simulations, which will improve our statistics and allow us to explore redshift trends more accurately.

With the upcoming large sky surveys and telescope missions, such as the James Webb Space Telescope (JWST; Gardner et al. 2006; Kalirai 2018), the MeerKAT Karoo Array Telescope HI Surveys (Holwerda et al. 2012), the Australian Square Kilometre Array Pathfinder HI surveys (Johnston et al. 2007, 2008), the Multi-Unit Spectroscopic Explorer (MUSE; Bacon et al. 2010), the K-band Multi-Object Spectrograph (KMOS; Wisnioski et al. 2015) surveys, and Atacama Large Millimeter/submillimeter Array (ALMA) future surveys, we expect to be able to study ring galaxies at higher redshifts and larger volumes due to their deep and large sky coverage. Through these surveys, we will also be able to test the simulation predictions and probe both the gas-phase pressure and the metallicity in these galaxies. This will further advance our understanding of the ISM as well as the star formation law in extreme collision cases.

\section{ACKNOWLEDGEMENTS}

We thank the referee, Curt Struck, for his comments which significantly improved the presentation of this manuscript. We also thank Joop Schaye for useful comments on the manuscript, and David Algorry for providing the list of barred galaxies identified in EAGLE and published in Algorry et al. (2017). AE wishes to Acknowledge the funds he received from the International Centre of Radio Astronomy (ICRAR). CL is funded by an Australian Research Council Discovery Early Career Researcher Award (DE150100618) and by the Australian Research Council Centre of Excellence for All Sky Astrophysics in 3 Dimensions (ASTRO 3D), through project number CE170100013. MS is supported by VENI grant 639.041.749. We acknowledge the Virgo Consortium for making their simulation data available. The EAGLE simulations were performed using the DiRAC-2 facility at Durham, managed by the ICC, and the PRACE facility Curie based in France at TGCC, CEA, Bruyeresle-Chatel. This work used the DiRAC Data Centric system at Durham University, operated by the Institute for Computational Cosmology on behalf of the STFC DiRAC HPC 
Facility (www.dirac.ac.uk). This equipment was funded by BIS National E-infrastructure capital grant ST/K00042X/1, STFC capital grant ST/H008519/1, and STFC DiRAC Operations grant ST/K003267/1 and Durham University. DiRAC is part of the National E-Infrastructure.

\section{REFERENCES}

Abraham R. G., van den Bergh S., Glazebrook K., Ellis R. S., Santiago B. X., Surma P., Griffiths R. E., 1996, ApJS, 107, 1

Algorry D. G., et al., 2017, MNRAS, 469, 1054

Appleton P. N., Marston A. P., 1997, AJ, 113, 201

Appleton P. N., Struck-Marcell C., 1987, ApJ, 312, 566

Appleton P. N., Struck-Marcell C., 1996, Fundamentals Cosmic Phys., 16, 111

Athanassoula E., Puerari I., Bosma A., 1997, MNRAS, 286, 284

Bacon R., et al., 2010, in Ground-based and Airborne Instrumentation for Astronomy III. p. 773508, doi:10.1117/12.856027

Baes M., et al., 2003, MNRAS, 343, 1081

Baes M., Verstappen J., De Looze I., Fritz J., Saftly W., Vidal Pérez E., Stalevski M., Valcke S., 2011, ApJS, 196, 22

Bahé Y. M., et al., 2016, MNRAS, 456, 1115

Baillard A., et al., 2011, A\&A, 532, A74

Barnes J. E., Hernquist L., 1996, ApJ, 471, 115

Blitz L., Rosolowsky E., 2006, ApJ, 650, 933

Bluck A. F. L., Conselice C. J., Buitrago F., Grützbauch R., Hoyos C., Mortlock A., Bauer A. E., 2012, ApJ, 747, 34

Bruzual G., Charlot S., 2003, MNRAS, 344, 1000

Buta R. J., 2017, MNRAS, 471, 4027

Camps P., Baes M., 2015, Astronomy and Computing, 9, 20

Camps P., Trayford J. W., Baes M., Theuns T., Schaller M., Schaye J., 2016, MNRAS, 462, 1057

Conn B. C., Fogarty L. M. R., Smith R., Candlish G. N., 2016, ApJ, 819, 165

Crain R. A., et al., 2009, MNRAS, 399, 1773

Crain R. A., et al., 2015, MNRAS, 450, 1937

Crain R. A., et al., 2017, MNRAS, 464, 4204

Cullen L., Dehnen W., 2010, MNRAS, 408, 669

D’Onghia E., Mapelli M., Moore B., 2008, MNRAS, 389, 1275

Dalla Vecchia C., Schaye J., 2012, MNRAS, 426, 140

Doi M., et al., 2010, AJ, 139, 1628

Durier F., Dalla Vecchia C., 2012, MNRAS, 419, 465

Elagali A., Wong O. I., Oh S.-H., Staveley-Smith L., Koribalski B. S., Bekki K., Zwaan M., 2018, MNRAS, 476, 5681

Elmegreen B. G., 1989, ApJ, 338, 178

Elmegreen B. G., 1993, ApJ, 411, 170

Elmegreen D. M., Elmegreen B. G., 2006, ApJ, 651, 676

Elmegreen B. G., Parravano A., 1994, ApJ, 435, L121

Fakhouri O., Ma C.-P., Boylan-Kolchin M., 2010, MNRAS, 406, 2267

Few J. M. A., Madore B. F., 1986, MNRAS, 222, 673

Fiacconi D., Mapelli M., Ripamonti E., Colpi M., 2012, MNRAS, 425, 2255

Fogarty L., et al., 2011, MNRAS, 417, 835

Furlong M., et al., 2015, MNRAS, 450, 4486

Furlong M., et al., 2017, MNRAS, 465, 722

Gardner J. P., et al., 2006, Space Sci. Rev., 123, 485

Gerber R. A., Lamb S. A., Balsara D. S., 1996, MNRAS, 278, 345

Giavalisco M., et al., 2004, ApJ, 600, L103

Gnedin N. Y., Kravtsov A. V., 2011, ApJ, 728, 88

Groves B., Dopita M. A., Sutherland R. S., Kewley L. J., Fischera J., Leitherer C., Brandl B., van Breugel W., 2008, ApJS, 176, 438

Hernquist L., Weil M. L., 1993, MNRAS, 261, 804

Herrera-Endoqui M., Díaz-García S., Laurikainen E., Salo H., 2015, A\&A, 582, A86

Higdon J. L., 1995, ApJ, 455, 524

Higdon J. L., 1996, ApJ, 467, 241
Higdon J. L., Higdon S. J. U., 2010, in Smith B., Higdon J., Higdon S., Bastian N., eds, Astronomical Society of the Pacific Conference Series Vol. 423, Galaxy Wars: Stellar Populations and Star Formation in Interacting Galaxies. p. 12 (arXiv: 1003.4691 )

Higdon J. L., Wallin J. F., 1997, ApJ, 474, 686

Higdon J. L., Higdon S. J. U., Rand R. J., 2012, ApJ, 756, 196

Higdon J. L., Higdon S. J. U., Martín Ruiz S., Rand R. J., 2015, ApJ, 814, L1

Holwerda B. W., Blyth S.-L., Baker A. J., 2012, in Tuffs R. J., Popescu C. C., eds, IAU Symposium Vol. 284, The Spectral Energy Distribution of Galaxies - SED 2011. pp 496-499 (arXiv:1109.5605), doi:10.1017/S1743921312009702

Hopkins P. F., 2013, MNRAS, 428, 2840

Hopkins P. F., Kereš D., Oñorbe J., Faucher-Giguère C.-A., Quataert E., Murray N., Bullock J. S., 2014, MNRAS, 445, 581

Hopkins P. F., et al., 2017, preprint, (arXiv: 1702.06148)

Horellou C., Combes F., 2001, Ap\&SS, 276, 1141

Hubble E. P., 1926, ApJ, 64

Ji I., Peirani S., Yi S. K., 2014, A\&A, 566, A97

Jiang C. Y., Jing Y. P., Faltenbacher A., Lin W. P., Li C., 2008, ApJ, 675, 1095

Johnston S., et al., 2007, Publ. Astron. Soc. Australia, 24, 174

Johnston S., et al., 2008, Experimental Astronomy, 22, 151

Kalirai J., 2018, Contemporary Physics, 59, 251

Katsianis A., et al., 2017, MNRAS, 472, 919

Kennicutt Jr. R. C., 1998, ApJ, 498, 541

Lagos C. D. P., Baugh C. M., Lacey C. G., Benson A. J., Kim H.-S., Power C., 2011, MNRAS, 418, 1649

Lagos C. d. P., et al., 2015, MNRAS, 452, 3815

Lagos C. d. P., et al., 2016, MNRAS, 459, 2632

Lagos C. d. P., et al., 2018, MNRAS, 473, 4956

Lavery R. J., Remijan A., Charmandaris V., Hayes R. D., Ring A. A., 2004, ApJ, 612, 679

Leroy A., Bolatto A. D., Simon J. D., Blitz L., 2005, ApJ, 625, 763

Leroy A. K., Walter F., Brinks E., Bigiel F., de Blok W. J. G., Madore B., Thornley M. D., 2008, AJ, 136, 2782

Lynds R., Toomre A., 1976, ApJ, 209, 382

Madore B. F., Nelson E., Petrillo K., 2009, ApJS, 181, 572

Man A. W. S., Zirm A. W., Toft S., 2016, ApJ, 830, 89

Mapelli M., Mayer L., 2012, MNRAS, 420, 1158

Mapelli M., Moore B., Giordano L., Mayer L., Colpi M., Ripamonti E., Callegari S., 2008a, MNRAS, 383, 230

Mapelli M., Moore B., Ripamonti E., Mayer L., Colpi M., Giordano L., 2008b, MNRAS, 383, 1223

Marinacci F., et al., 2017, preprint, (arXiv:1707.03396)

Mayya Y. D., Bizyaev D., Romano R., Garcia-Barreto J. A., Vorobyov E. I., 2005, ApJ, 620, L35

McAlpine S., et al., 2016, Astronomy and Computing, 15, 72

Mihos J. C., Hernquist L., 1994, ApJ, 437, 611

Naab T., Khochfar S., Burkert A., 2006, ApJ, 636, L81

Naab T., et al., 2014, MNRAS, 444, 3357

Naiman J. P., et al., 2017, preprint, (arXiv:1707.03401)

Nair P. B., Abraham R. G., 2010, ApJS, 186, 427

Nelson D., et al., 2017, preprint, (arXiv:1707.03395)

Parker Q. A., Zijlstra A. A., Stupar M., Cluver M., Frew D. J., Bendo G., Bojičić I., 2015, MNRAS, 452, 3759

Pillepich A., et al., 2017, preprint, (arXiv:1707.03406)

Planck Collaboration et al., 2014, A\&A, 571, A16

Price D. J., 2008, Journal of Computational Physics, 227, 10040

Qu Y., et al., 2017, MNRAS, 464, 1659

Rahmati A., Pawlik A. H., Raičević M., Schaye J., 2013, MNRAS, 430, 2427

Renaud F., et al., 2018, MNRAS, 473, 585

Rix H.-W., et al., 2004, ApJS, 152, 163

Rodriguez-Gomez V., et al., 2017, MNRAS, 467, 3083

Romano R., Mayya Y. D., Vorobyov E. I., 2008, AJ, 136, 1259

Rosas-Guevara Y. M., et al., 2015, MNRAS, 454, 1038 
Schaller M., Dalla Vecchia C., Schaye J., Bower R. G., Theuns T., Crain R. A., Furlong M., McCarthy I. G., 2015, MNRAS, 454, 2277

Schaye J., 2004, ApJ, 609, 667

Schaye J., Dalla Vecchia C., 2008, MNRAS, 383, 1210

Schaye J., et al., 2010, MNRAS, 402, 1536

Schaye J., et al., 2015, MNRAS, 446, 521

Smith R., Lane R. R., Conn B. C., Fellhauer M., 2012, MNRAS, 423, 543

Snyder G. F., et al., 2015, MNRAS, 454, 1886

Springel V., 2005, MNRAS, 364, 1105

Springel V., et al., 2008, MNRAS, 391, 1685

Springel V., et al., 2017, preprint, (arXiv:1707.03397)

Straatman C. M. S., et al., 2016, ApJ, 830, 51

Struck C., 2010, MNRAS, 403, 1516

Struck-Marcell C., Lotan P., 1990, ApJ, 358, 99

Theys J. C., Spiegel E. A., 1977, ApJ, 212, 616

Toomre A., 1977, in Tinsley B. M., Larson D. Campbell R. B. G., eds, Evolution of Galaxies and Stellar Populations. p. 401

Trayford J. W., et al., 2015, MNRAS, 452, 2879

Trayford J. W., Theuns T., Bower R. G., Crain R. A., Lagos C. d. P., Schaller M., Schaye J., 2016, MNRAS, 460, 3925

Trayford J. W., et al., 2017, MNRAS, 470, 771

Weinberger R., et al., 2017, MNRAS, 465, 3291

Wendland H., 1995, Advances in Computational Mathematics, 4, 389

White S. D. M., 1978, MNRAS, 184, 185

Wiersma R. P. C., Schaye J., Smith B. D., 2009a, MNRAS, 393, 99

Wiersma R. P. C., Schaye J., Theuns T., Dalla Vecchia C., Tornatore L., 2009b, MNRAS, 399, 574

Willett K. W., et al., 2013, MNRAS, 435, 2835

Wisnioski E., et al., 2015, ApJ, 799, 209

Wong O. I., et al., 2006, MNRAS, 370, 1607

Wong O. I., et al., 2017, MNRAS, 466, 574

de Vaucouleurs G., 1959, Handbuch der Physik, 53, 275

\section{APPENDIX A: LIST OF EAGLE RING GALAXIES}

Table A1 lists all the ring galaxies identified in the EAGLE simulations up until redshift $z=2.23$.

This paper has been typeset from a $\mathrm{T}_{\mathrm{E}} \mathrm{X} / \mathrm{L} \mathrm{T}_{\mathrm{E}} \mathrm{X}$ file prepared by the author.
Table A1. Ring galaxies identified in the EAGLE simulation.

\begin{tabular}{|c|c|c|c|c|}
\hline GalaxyID & Snapshot & $\mathrm{z}$ & Group N. & Subgroup N. \\
\hline 1419938 & & & 164 & 1 \\
\hline 13176886 & & & 63 & 2 \\
\hline 14042156 & & & 130 & 1 \\
\hline 15358400 & 28 & 0 & 258 & 0 \\
\hline 15511663 & & & 281 & 0 \\
\hline 15978820 & & & 342 & 0 \\
\hline 18010353 & & & 841 & 0 \\
\hline 16736005 & & & 501 & 0 \\
\hline 16750450 & & & 467 & 0 \\
\hline 14042157 & & & 465 & 0 \\
\hline 11525816 & 27 & 0.10 & 15 & 5 \\
\hline 13839082 & & & 181 & 2 \\
\hline 8903544 & & & 1174 & 0 \\
\hline 15476546 & & & 281 & 0 \\
\hline 20755891 & & & 120 & 0 \\
\hline 8930054 & & & 1232 & 0 \\
\hline 9078223 & & & 994 & 0 \\
\hline 13176888 & 26 & 0.18 & 62 & 1 \\
\hline 14681036 & & & 206 & 0 \\
\hline 16832146 & & & 32 & 1 \\
\hline 18010355 & & & 841 & 0 \\
\hline 18010356 & & & 842 & 0 \\
\hline 16347102 & & & 532 & 0 \\
\hline 13825639 & 25 & 0.27 & 164 & 2 \\
\hline 20629170 & & & 128 & 0 \\
\hline 13660663 & & & 76 & 2 \\
\hline 15978824 & & & 540 & 0 \\
\hline 16229689 & & & 668 & 0 \\
\hline 16482632 & 24 & 0.36 & 432 & 0 \\
\hline 16921472 & & & 630 & 0 \\
\hline 17451600 & & & 519 & 0 \\
\hline 17961354 & & & 557 & 0 \\
\hline 14418325 & & & 473 & 0 \\
\hline 15187269 & & & 845 & 0 \\
\hline 16482633 & 23 & 0.50 & 478 & 0 \\
\hline 15511668 & & & 342 & 0 \\
\hline 18026177 & & & 888 & 0 \\
\hline 15978825 & & & 569 & 0 \\
\hline 20259289 & & & 107 & 0 \\
\hline 18078238 & & & 1133 & 0 \\
\hline 15528603 & 22 & 0.61 & 477 & 0 \\
\hline 16521451 & & & 500 & 0 \\
\hline 15528604 & & & 476 & 0 \\
\hline 15289527 & 21 & 0.73 & 403 & 0 \\
\hline 8931586 & & & 1333 & 0 \\
\hline 15528605 & 20 & 0.86 & 531 & 0 \\
\hline 16701972 & 19 & 1.0 & 913 & 0 \\
\hline 14371833 & 19 & 1.0 & 130 & 0 \\
\hline 9135999 & 19 & 1.0 & 1805 & 0 \\
\hline 16701973 & 18 & 1.26 & 965 & 0 \\
\hline 17643587 & 18 & 1.26 & 905 & 0 \\
\hline 17643588 & 17 & 1.48 & 828 & 0 \\
\hline 19899640 & 17 & 1.48 & 352 & 0 \\
\hline 20640573 & 16 & 1.73 & 233 & 0 \\
\hline 20727481 & 14 & 2.23 & 780 & 0 \\
\hline
\end{tabular}

